\title{
Haches et herminettes en pierre de Guyane française
}

Stéphen Rostain, Yves Wack

\section{Citer ce document / Cite this document :}

Rostain Stéphen, Wack Yves. Haches et herminettes en pierre de Guyane française. In: Journal de la Société des Américanistes. Tome 73, 1987. pp. 107-138;

doi : https://doi.org/10.3406/jsa.1987.1026

https://www.persee.fr/doc/jsa_0037-9174_1987_num_73_1_1026

Fichier pdf généré le 05/05/2018 


\section{Résumé}

Until now, few archaeologists have studied stone tools that are essential for Amerindian life, and in particular axes and adzes. In French Guiana, most lithic objects are polished and classed according to morphologic and dimensional type. Amérindien craftsmen took great care in choosing their rocks and various techniques were used to put polished stones into wooden handles. Mostly used for wood carving and defense, axes and adzes also had cultual and symbolic value. With the exception of several regional types, the sames blades are found throughout the Amazon, Guianas and the Caribbean. The stone axe is still used by rare Amerindian groups and certains attach legends to their use. Archaeological study has just begun on this new field.

\section{Resumen}

Pocos arqueólogos han estudiado hoy en dia las herramientas de piedra a pesar que estas, particularmente las hachas y las azuelas, son esenciales al indigena sudamericano para la vida en la selva. En Guayana francesa, la mayor parte del material litico conocido esta pulido. Ha sido clasificado según una tipología morfológica y dimensional. Las rocas utilizadas por el artesano indigena para la manufactura de hachas y azuelas sugiere una selección minuciosa. Después de haber pulido la piedra sobre una roca se le hacia un mango de maděra, según diversas técnicas. Estos instrumentes se usan principalmente para trabajar la maděra y como armamento pero tienen igualmente una función simbólica y cultual. Con exception de algunas variantes régionales, los mismos tipos de cuchilla se encuentran en Amazonia, en las Guayanas y en las Antillas. Utilizada hoy por pocos grupos amerindios y objeto de leyenda para algunos, el estudio del hacha de piedra comienza a interesar a los arqueológos abriendo un campo de investigaciones prometedor. 


\title{
HACHES ET HERMINETTES EN PIERRE DE GUYANE FRANÇAISE
}

\author{
Stéphen ROSTAIN * \\ et \\ Yves $\mathrm{WACK} * *$ \\ "Ces gens (les Amérindiens) passent de longs \\ moments à lisser leur outil ».
}

Abbé Périgny (xvIII siècle).

Peu d'archéologues ont à ce jour étudié les outils en pierre bien que ceux-ci --.. et tout particulièrement les haches et les herminettes -.. eussent été essentiels à l'Amérindien pour la vie en forêt.

En Guyane française, la plus grande part du matériel lithique connu est poli. Il est classé selon une typologie morphologique et dimensionnelle.

La nature des roches utilisées suggère un choix pétrographique minutieux de la part des artisans amérindiens. La pierre, après un polissage sur un rocher, était ensuite généralement emmanchée dans un bois, selon diverses techniques.

Les haches et les herminettes ont pour principales fonctions le travail et l'armement, mais également des destinations symboliques ou cultuelles.

Exception faite de quelques types régionaux, les mêmes types de lames se retrouvent en Amazonie, dans toutes les Guyanes et sur les Antilles.

Encore utilisée par de rares groupes amérindiens, en même temps qu'objet de légendes pour d'aucuns, la hache de pierre commence seulement à être étudiée et ouvre à l'archéologue un champ d'investigation prometteur.

Hachas y azuelas de piedra de la Guayana francesa.

Pocos arqueólogos han estudiado hoy en dia las herramientas de piedra a pesar que éstas, particularmente las hachas y las azuelas, son esenciales al indigena sudamericano para la vida en la selva.

En Guayana francesa, la mayor parte del material lítico conocido está pulido. Ha sido clasificado según una tipología morfológica y dimensional.

Las rocas utilizadas por el artesano indigena para la manufactura de hachas y azuelas sugiere una selección minuciosa. Después de haber pulido la piedra sobre una roca se le hacía un mango de madera, según diversas técnicas.

* Centre de Recherche d'Archéologie Précolombienne de l'Université de Paris I, Panthéon-Sorbonne.

** Lycée Félix-Éboué, Cayenne.

J.S.A. 1987, LXXIII : p. 107 à 138. 


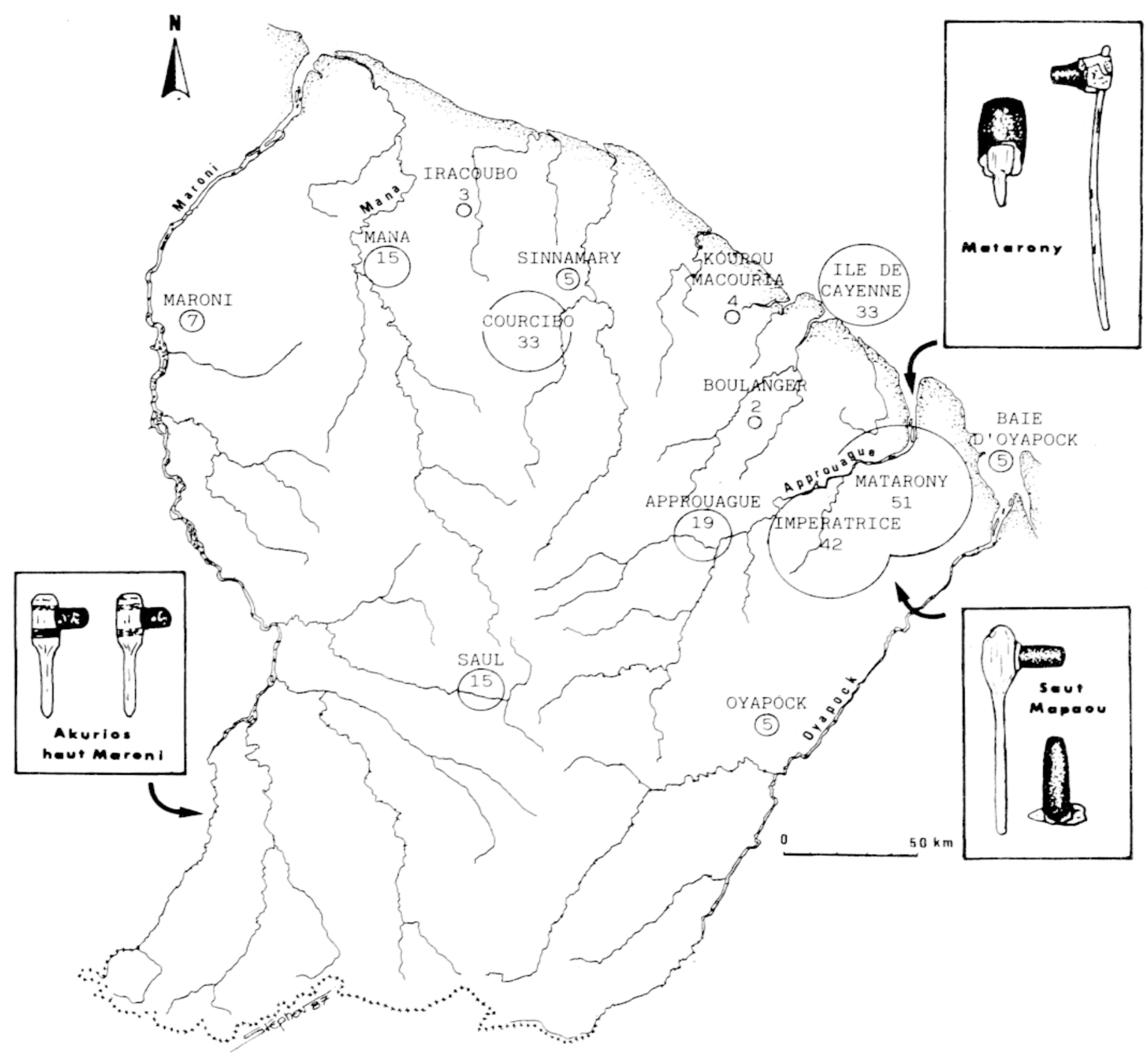

Fig. 1. - Localisation géographique de la découverte des haches et herminettes en pierre de Guyane française. Parmi les 239 pièces localisées 37 proviennent de sites archéologiques prospectés ou fouillés. 
Estos instrumentos se usan principalmente para trabajar la madera y como armamento pero tienen igualmente una función simbólica y cultual.

Con excepción de algunas variantes regionales, los mismos tipos de cuchilla se encuentran en Amazonia, en las Guayanas y en las Antillas.

Utilizada hoy por pocos grupos amerindios y objeto de leyenda para algunos, el estudio del hacha de piedra comienza a interesar a los arqueológos abriendo un campo de investigaciones prometedor.

\section{Stone axes and adzes of French Guiana.}

Until now, few archaeologists have studied stone tools that are essential for Amerindian life, and in particular axes and adzes.

In French Guiana, most lithic objects are polished and classed according to morphologic and dimensional type.

Amerindien craftsmen took great care in choosing their rocks and various techniques were used to put polished stones into wooden handles.

Mostly used for wood carving and defense, axes and adzes also had cultual and symbolic value.

With the exception of several regional types, the sames blades are found throughout the Amazon, Guianas and the Caribbean.

The stone axe is still used by rare Amerindian groups and certains attach legends to their use. Archaeological study has just begun on this new field.

La hache en pierre fut un outil essentiel à l'Amérindien pour la vie en forêt, aussi retrouve-t-on un outillage en pierre abondant dans les sites archéologiques des Guyanes. Cependant, la division chronologique entre haches taillées et haches polies telle qu'elle est définie en Europe est difficile à appliquer au continent américain, puisqu'il semble que les deux types aient ici coexisté. En outre, les travaux archéologiques en Guyane étant fort jeunes et à un stade encore embryonnaire, les sites étudiés n'ont pas été attribués à des époques précises, ni à des groupes amérindiens particuliers. Aussi les conclusions de cet article serontelles principalement basées sur les techniques de fabrication des haches et des herminettes (choix des roches, façonnage de la lame, emmanchement), leur morphologie et leurs différentes utilisations.

Ce travail de recherche sur la pierre chez les Amérindiens a commencé au début de l'année 1986. Il a tout d'abord consisté en un répertoriage, sur fiches de renseignements individuelles, de collections privées d'outils lithiques. Une recherche bibliographique d'anciens récits de voyageurs ainsi que d'études sur le matériel amérindien en pierre a précédé l'analyse des données. Les informations présentées ici en sont les premiers résultats, mais d'autres hypothèses demeurent en cours d'étude et de vérification. 


\section{LES TRAVAUX PRÉCÉDENTS SUR LES OUTILS EN PIERRE}

L'intérêt pour les haches en pierre des Guyanes est récent puisqu'il ne remonte qu'au début de ce siècle. Walter Roth fut le premier à étudier ce type de matériel. En 1924, il publiait une description des différents instruments utilisés autrefois par les Amérindiens et proposait une typologie.

Trente ans plus tard, Henry et Paule Reichlen décrivaient les collections d'outils lithiques de Guyane française, entreposées au Musée de l'Homme à Paris. Plus récemment, Aad Boomert a également travaillé sur le matériel en pierre des Guyanes et a écrit deux articles fondamentaux en 1977 et en 1979 : le premier est une analyse pétrographique et géographique des outils en pierre du Surinam à partir de laquelle l'auteur déduit certaines relations commerciales entre différents groupes amérindiens. Dans le second article, il classe 600 à 700 pièces des Guyanes selon une typologie morphologique très détaillée.

Hormis ces auteurs, peu de chercheurs ont étudié l'outillage amérindien des Guyanes. Pour l'aire Caraïbe, Peter Harris a publié en 1983 une longue étude typologique sur les haches des Antilles. Enfin, on trouve également quelques petits articles ponctuels sur le sujet.

Ce domaine de la recherche archéologique dans l'aire amazonienne est donc encore quasiment inconnu. Pourtant, l'étude des instruments en pierre pourrait aider à mieux comprendre les conditions de vie des anciens occupants de ce territoire, et ses premières conclusions ouvrent des perspectives prometteuses.

\section{LA TYPOLOGIE DES HACHES ET HERMINETTES}

\section{La typologie morphologique}

Sur plus de 500 outils en pierre observés lors de cette recherche, 372 pièces ont été répertoriées individuellement. Parmi celles-ci, 278 sont des haches, des herminettes ou des ciseaux (comprenant 17 fragments trop petits pour être classés dans un type précis), tandis que les autres sont des instruments divers : pilons, broyeurs, percuteurs, " marteaux ", " boules "... dont l'étude est en cours de réalisation.

La typologie des haches en pierre polie élaborée par Aad Boomert (1979) sur les Guyanes est actuellement la plus complète et la plus précise qui existe. Elle est principalement basée sur la forme des talons et des tranchants des haches. Le critère de distinction choisi - la morphologie du talon - correspond apparemment à la méthode d'emmanchement de la lame. Le trait majeur de différenciation est la présence d'un tranchant simple ou double. Chacun de ces deux types se scindent en plusieurs groupes. Parmi les haches à tranchant unique, Aad Boomert en distingue cinq : haches simples, les haches à oreilles, les haches à encoches et les haches gravées ou sculptées (ces deux derniers types n'étant apparemment pas 


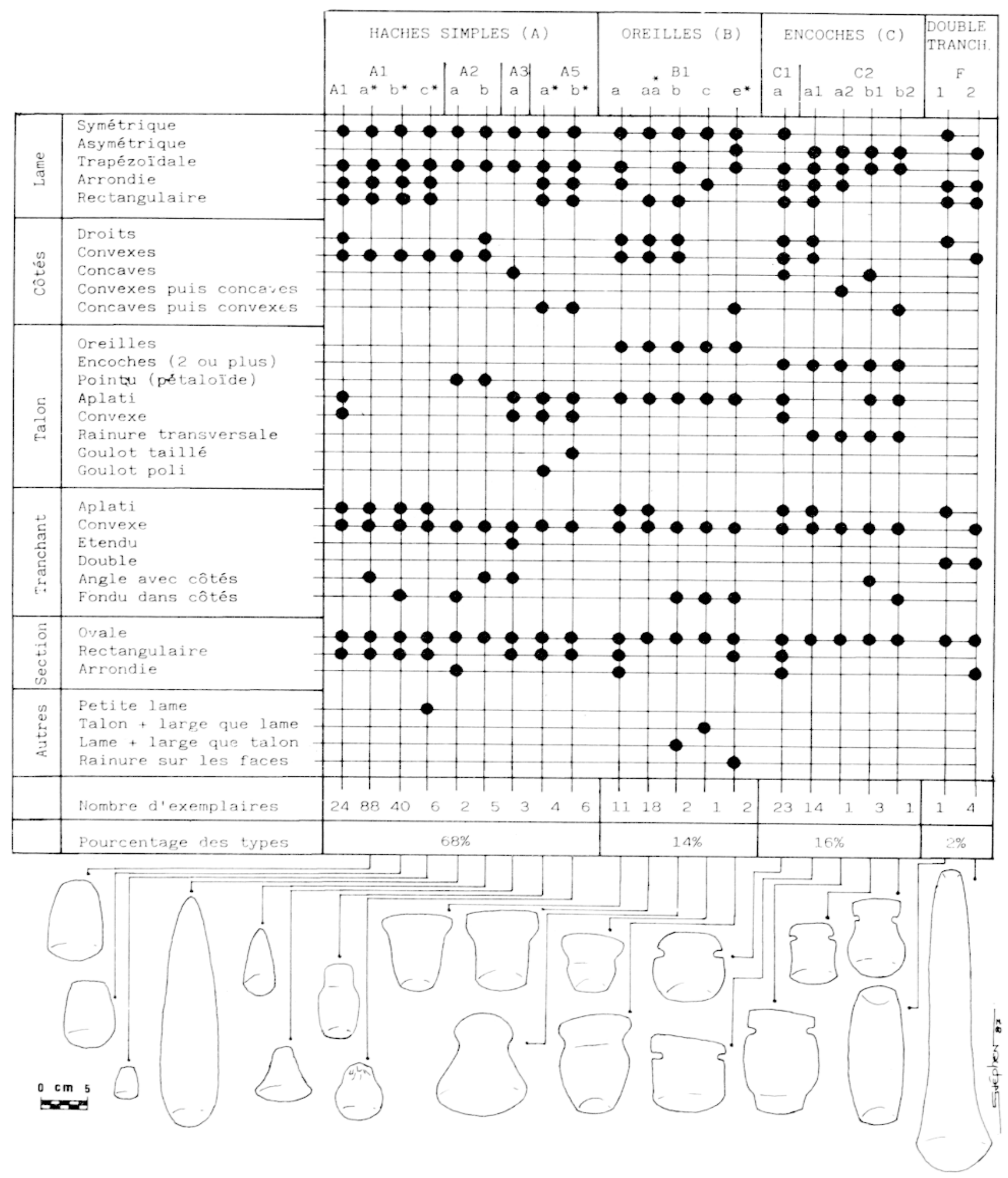

Fig. 2. -- Tableau typologique de la morphologie des haches et herminettes de Guyane française revu d’après la typologie de Aad Boomert (1979), 261 pièces (les astérisques correspondent aux nouveaux types déterminés pour la Guyane française). 
représentés en Guyane française). Dans chaque groupe, existent encore des subdivisions en fonction de la forme de la lame.

Un tableau typologique des caractéristiques morphologiques déterminées par Aad Boomert, auxquelles s'adjoignent de nouveaux types, reprend le classement proposé : en abscisse, les codes de chaque type, et en ordonnée, leurs caractéristiques (figure 2).

Les haches simples sont divisées en quatre types majeurs réunissant eux-mêmes huit sous-groupes, les haches à oreilles en cinq sous-groupes, les haches à encoches en deux types majeurs comprenant cinq sous-groupes. Enfin, les haches à double tranchants sont de deux types différents.

La lecture horizontale du tableau fait apparaître une nette précominance de haches symétriques, trapézoïdales, à tranchant convexe et à section ovale. Verticalement, on voit dominer le nombre de haches simples $(68 \%)$ sur la quantité de haches à oreilles et de haches à encoches, ces deux types constituant à peu près le même pourcentage de l'échantillon (15\%). Quant aux haches à double tranchant, elles sont très nettement plus rares $(2 \%)$.

Établie seulement à partir de la morphologie des outils, cette typologie permet un classement pratique des haches et des herminettes. En revanche, elle demeure assez limitée et ne met pas en évidence des données suffisantes pour l'étude de la fabrication et de l'utilisation des instruments. On pourrait envisager de créer une classification des difficultés de fabrication d'un outil : le polissage d'un côté concave serait alors le plus compliqué. A partir de l'échantillon étudié ici, de nouvelles typologies, basées sur certains calculs d'indices dimensionnels, ont été tentées.

\section{L'analyse statistique}

L'échantillon de haches et d'herminettes étudiées comporte 274 exemplaires, sur lesquels ont été mesurés les paramètres longueur (L), largeur (1), épaisseur (e), longueur du tranchant $(\mathrm{Lt})$ et poids $(\mathrm{P})$. A l'aide de ces paramètres, peuvent être calculés certains indices comme la dimension moyenne, en centimètres, $(\mathrm{L}+1+$ e) $/ 3$, l'indice d'aplatissement, sans dimension, $(L+1) / 2 e$ (ces deux indices sont utilisés en géomorphologie, Perriaux 1967), et enfin l'indice de percussion, en grammes par centimètre, $\mathbf{P} / \mathbf{L t}$.

Pour l'échantillon des 274 haches, tous les paramètres cités ci-dessus n'ont pu être définis. Ainsi, les échantillons réellement étudiés sont de 217 pour la dimension moyenne et l'indice d'aplatissement, et de 174 pour l'indice de percussion. Dans les deux premiers cas, il existe environ une soixantaine de fragments sur lesquels une des trois dimensions au moins n'a pu être mesurée. Dans le dernier cas, ce sont 100 mesures de poids qui n'ont pu être réalisées. Ces trois critères d'analyse et le paramètre poids ont été classés par ordre décroissant.

La "Dimension Moyenne " (figure 3a) des échantillons est comprise entre 2 et $12 \mathrm{~cm}$, avec une moyenne de $6,7 \mathrm{~cm}$. On notera une hache à double tranchant mesurant $36 \mathrm{~cm}$ de long (EL6), qui se distingue par sa grande dimension; elle est également la pièce la plus lourde avec $1.910 \mathrm{~g}$ (figure $11 \mathrm{~d}$ ). Le mode, la médiane et la moyenne sont de valeurs voisines. L'échantillon se répartit selon une loi 
Gaussique classique (courbe en cloche), ce qui n'est pas trop surprenant compte tenu de la définition de ce critère. Celui-ci a le mérite de montrer qu'il s'agit bien des mêmes pièces et d'une population relativement bien répartie.

"L'Indice d'Aplatissement " (figure 3b), sans dimension, permet de mieux préciser la morphologie des objets lithiques: en effet, selon sa définition, plus faible est l'épaisseur, plus l'indice est grand. Cet indice est compris entre 1 et 7 avec une moyenne de 3,3. La classe 2 est la plus abondante (100 valeurs). Ceci montre que les pièces sont dans l'ensemble plutôt épaisses. Seules 15 haches garnissent les classes d'indices 5, 6 et 7 . La répartition est asymétrique et une faible partie de l'échantillon est peu épaisse.

a) Dimension moyenne, $(\mathrm{L}+1+\mathrm{e}) / 3$.

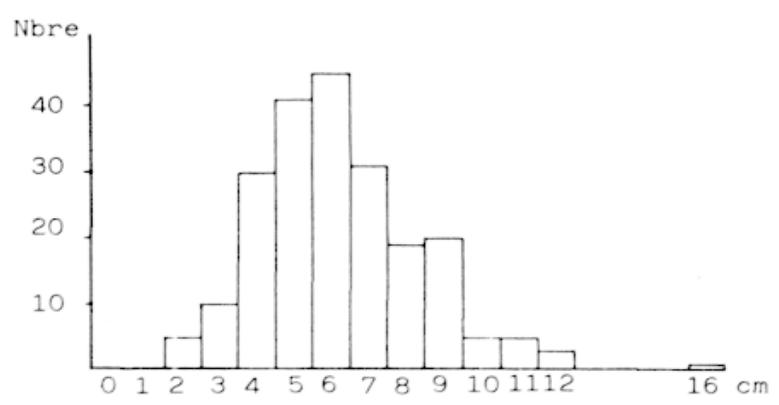

b) Indice d'aplatissement, $(\mathrm{L}+1) / 2 \mathrm{e}$. Nbre

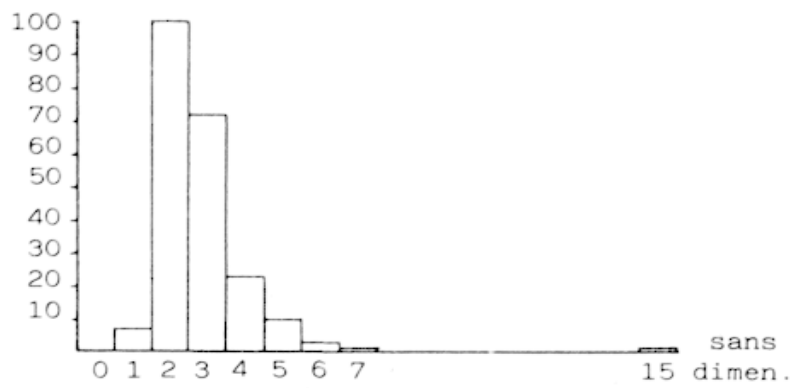

c) Poids.
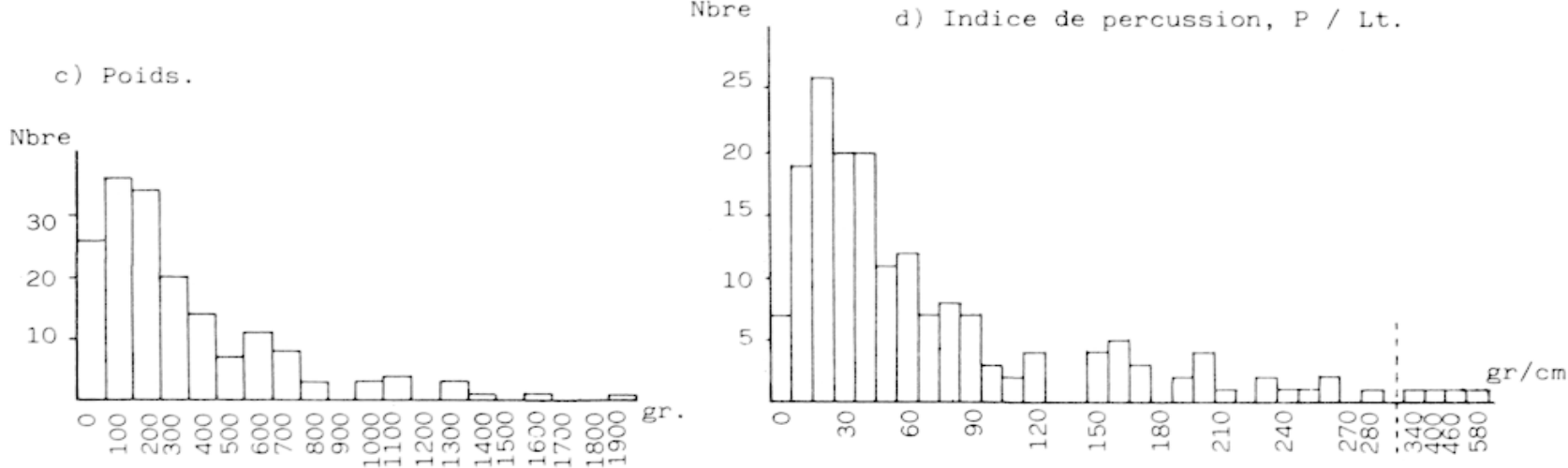

e) Tableau récapitulatif de quelques paramètres statistiques:

\begin{tabular}{|c|c|c|c|c|c|c|}
\hline VARIABLE & NOMBRE & MODE & MEDIANE & MOYENNE & ECART-TYPE & OBSERVATIONS \\
\hline Dim. moy.cm & 217 & 6,0 & 6,3 & 6,7 & 2,14 & répartition quasi Gaussique \\
\hline Indice apl. & 217 & 2,0 & 3,0 & 3,3 & 1,24 & répartition quasi Gaussique \\
\hline Poids.g & 217 & 100 & 250 & 376 & 344 & asymètrique \\
\hline Indi. perc. \\
\hline g/cm
\end{tabular}

- Mode: valeur correspondant au plus grand effectif dans une classe.

- Médiane: valeur rencontrée au centre de l'échantilion classé.

- Moyenne: valeur moyenne de l'échantilion (Somme valeurs) / Nombre.

- Ecart-Type: racine carrée de la fluctuaction ou variance (moyenne des carrés des écarts à la moyenne).

FiG. 3. - Histogrammes et tableau de synthèse de l'analyse statistique des haches en pierre. 
Cet indice met en évidence l'exceptionnelle minceur de la hache taillée AL11 (figure 8d).

Si l'on ne considérait que les deux caractères morphologiques pré-cités, on pourrait conclure à un échantillon assez homogène dans sa morphologie. Mais il est vrai que par la définition de ces deux critères - somme et rapports de longueur - on ne peut guère entrer dans une analyse plus fine qui mettrait éventuellement en évidence divers types de haches.

Le paramètre "Poids " (figure 3c) semble suivre la répartition obtenue sur le classement des indices d'aplatissement, augmenté d'un cortège de haches $(20 \%)$ plus lourdes que la moyenne (376 g) et comprises entre $600 \mathrm{~g}$ et $1.900 \mathrm{~g}$. Cette mesure rend mieux compte du type de population étudiée. On pourrait voir deux groupes d'outils :

Le premier, compris entre $10 \mathrm{~g}$ et $400 \mathrm{~g}$, et représentant $80 \%$ de l'échantillon, est constitué de petits objets de masse moyenne $(250 \mathrm{~g})$.

Le second, plus faible en effectif, comporte une série de pièces nettement plus lourdes avec une prédominance vers les classes $700 \mathrm{~g}$ et $1.200 \mathrm{~g}$. Cette birépartition de l'échantillon explique la valeur élevée de l'écart-type.

"L'Indice de Percussion" (figure $3 \mathrm{~d}$ ), plus intéressant de notre point de vue pour l'étude de ces pièces, accuse mieux encore la répartition en deux populations différentes évoquée plus haut. Cet indice exprimé en $\mathrm{g} / \mathrm{cm}$, est compris entre 2,1 et 583. De par sa définition, cet indice aurait une parenté avec une pression (masse/surface).

La valeur de l'écart-type indique la grande dispersion de l'échantillon. En effet, $66 \%$ de l'ensemble se trouve relativement groupé vers les faibles valeurs de classe, c'est-à-dire entre 2 et $60 \mathrm{~g} / \mathrm{cm}$. Les $34 \%$ restant sont étalés dans les classes comprises entre 70 et $580 \mathrm{~g} / \mathrm{cm}$, avec un noyau d'une vingtaine de haches $(11,5 \%)$ autour de la classe $160 \mathrm{~g} / \mathrm{cm}$ (poids moyen $=700 \mathrm{~g}$ ).

Notons enfin que les forts indices ne correspondent pas forcément aux pièces les plus lourdes. C'est l'élément TN1 qui a le plus fort indice avec une masse de $1.400 \mathrm{~g}$, mais, dans la classe 400 (la troisième), on trouve une hache pesant seulement $490 \mathrm{~g}$. Cela signifie que certains tranchants très étroits permettent d'augmenter la force de percussion de l'outil.

C'est à travers l'étude de cet indice, qu'il faudrait pousser plus avant, que l'expression du polissage manuel devient plus évidente. Par opposition à un échantillon assez homogène de haches et d'herminettes de poids moyen de $200 \mathrm{~g}$ et d'un indice voisin de 30 , on observe un autre échantillon, nettement dispersé autour d'un poids moyen de $700 \mathrm{~g}$ et d'un indice de 160 .

Cette remarque met en évidence, de notre point de vue, deux populations de haches et d'herminettes polies et, par là-même, sans doute deux usages distincts. Seule une étude statistique plus poussée avec un échantillon plus large pourrait confirmer cette hypothèse. Mise en parallèle avec d'autres données, comme par exemple l'analyse des micro-traces et des micro-restes présents sur les tranchants, elle permettrait peut-être de différencier deux types d'outils, adaptés chacun à des fonctions précises. 


\section{LA FABRICATION DES OUTILS}

\section{Les choix pétrographiques}

Loin de prendre n'importe quelle pierre, les Amérindiens paraissent avoir choisi les roches destinées à être travaillées pour devenir objets utilitaires. On peut distinguer les instruments tranchants des outils non tranchants.

Les qualités recherchées. Pour obtenir un outil tranchant efficace, plusieurs qualités sont souhaitées: dureté, aptitude à l'abrasion régulière, résistance du tranchant, densité élevée, résistance à l'altération et peut-être également des qualités esthétiques.

Les pierres devaient avoir une dureté satisfaisante, mais permettant un façonnage ni trop difficile, ni trop long. Ainsi, les quartz (dureté 7), pourtant communs et présentant de forts indices d'aplatissement, ne sont qu'exceptionnellement choisis (un seul spécimen connu, provenant de Macouria, YA 2, figure 8a).

Les roches imprégnées presque exclusivement de silice colloïdale (jaspes) ou les verres sont de même exclus.

L'aptitude à l'abrasion régulière est indispensable pour des outils tranchants, et cette exigence avantage les roches homogènes.

Les phénomènes de silicification, fréquents dans les terrains métamorphisés de la série Paramaca, lorsqu'ils ne sont pas excessifs, s'avèrent favorables : en général, les grès fins, sous l'abrasion, voient se déloger régulièrement leurs grains hors du ciment siliceux qui les unit.

La résistance du fil tranchant est également primordiale. L'angle formé par les deux faces du biseau est une des principales limites des outils en pierre : 50 à $60^{\circ}$ en moyenne contre 10 à $20^{\circ}$ pour des outils en fer de même fonction. La raison en est que le fil du tranchant ne doit pas buter, à l'aiguisage, sur un débris plus épais. Les cristaux (comme les plagioclases) des roches grenues, possèdent des clivages, souvent nombreux et disposés en tous sens : ils se fractureraient, altérant le taillant. Les pyroxènes de gabbros, comme par exemple l'augite, offriraient une cassure en marche d'escalier. Si l'artisan peut exercer sa préférence, il ne recherchera pas les roches trop largement grenues, les grès grossiers ou les brèches, les roches vacuolaires comme les roches ferralitiques, les faciès ardoisiers ou schisteux, à cassure lamellaire.

Une densité élevée est indispensable pour que la force de percussion compense les limites du taillant de l'outil. Les roches magmatiques basiques (densité $>3$ ) sont ainsi avantagées. Les roches riches en pyroxènes, amphiboles notamment, conviennent bien, surtout si une silicification a pu compenser la dureté moindre de ces minéraux dont la densité est de 5 à 6 environ.

La résistance à l'altération est aussi considérée. Les roches contenant beaucoup de feldspaths sont sujettes à une altération (la kaolinisation) assez rapide : elles seront donc facilement écartées du choix pétrographique.

Enfin, certaines qualités " esthétiques " ont pu avoir leur importance, comme l'éclat et la couleur de la pierre. L'abondance de minéraux fibreux contribue à 
donner aux roches, après polissage, un éclat nacré et un toucher soyeux. Un ton vert, léger ou foncé, mêlé de gris ou de brun, prédomine dans la série étudiée, car les roches choisies contiennent des minéraux verts, fréquents dans les roches basiques ou ultrabasiques (le plagioclase andésine, la hornblende vert-foncé des andésites ou des micro-diorites, certains pyroxènes des gabbros, l'olivine des basaltes, eux-mêmes très rares par ailleurs).

Les roches effectivement sélectionnées. Sur les 58 outils tranchants étudiés en macroscopie, un large tiers appartient aux roches volcano-sédimentaires, brèches, grauwackes, et surtout $(32 \%)$ tufs fins ou cinérites silicifiées.

Les roches volcano-sédimentaires, comme les tufs fins ou les cinérites, ont été formées lors d'explosions volcaniques. Devenues partie intégrante du socle guyanais, ces roches en partagent l'évolution par métamorphisme ou par silicification. Bien homogène, sans cristaux apparents, modérément dur, coloré, susceptible de prendre un bel éclat et un poli onctueux, ce matériau semble avoir été le plus prisé par l'artisan néolithique amérindien.

Les roches magmatiques éruptives ou cristallines sont un des principaux constituants du sous-sol guyanais. Les laves acides ou basiques sont relativement peu représentées dans l'échantillonnage $\left(1 / 10^{\mathrm{e}}\right)$, en dépit de l'atout d'une densité élevée. Il en est de même pour les gabbros et les dolérites, pourtant également très denses. Les diorites et les granodiorites fournissent $17 \%$ du matériel total; les clivages nombreux de leurs feldspaths sont gênants pour un tranchant fin, mais leur abondance, l'urgence et la nécessité peuvent expliquer qu'elles aient été parfois utilisées.

La collecte des échantillons. Exigeants sur leur matériel, les artisans se heurtent à un problème de collecte car, si les massifs de schistes verts sont étendus, les affleurements sont rares et difficiles à voir. E. Aubert de la Rue remarquait en 1948 qu'ils étaient largement masqués par la végétation et par la couche épaisse d'altérites superficielles (argiles, alluvions latéritiques). Par exemple, dans la haute Comté, le Paramaca est souvent couronné de carapaces latéritiques et donne peu d'affleurements. En outre, les faciès changent vite et les beaux échantillons de tuf fin, de grande taille, sans diaclases ou schistosités, bien colorés, sont rares. L'idée de mine ou de carrière exploitée ponctuellement paraît plausible. Au Surinam, Aad Boomert et S. B. Kroonenberg (1977) ont montré comment, dans un de ces nombreux courants d'échange du monde amérindien, le Massif du Brownsberg aurait fourni des ébauches en métabasalte aux populations de la plaine côtière qui en était dépourvue. De Chabrillan, voyageant en 1742 sur le haut Camopi, remarque que "chacune de ces nations a son objet de commerce ". Il cite les Caicouianne; " gens du commerce des chiens de chasse ", les Ouen qui tirent leur nom des échanges de grages (rapes) à manioc qu'ils confectionnent en incrustant des éclats de silex sur des planches en bois. Goupy des Marets décrit également le commerce de pierres : « (...) les pierres vertes ce prennent fort haut dans la rivière des Amazones, que c'est des doucins quy se prennent dans le fon de cette rivière; que ces doucins sont de certaines vennes de limon (...) " (l'auteur fait ici allusion au jade du Minas Gerais utilisé pour des objets précieux, des figurines, des pendentifs...). Les cinérites recueillies dans les marmites torrentielles des sauts, issues des massifs de roches basiques métamorphorisées, ont pu être aussi au centre des trocs : on trouve des objets dans des zones où les roches vertes sont absentes, 

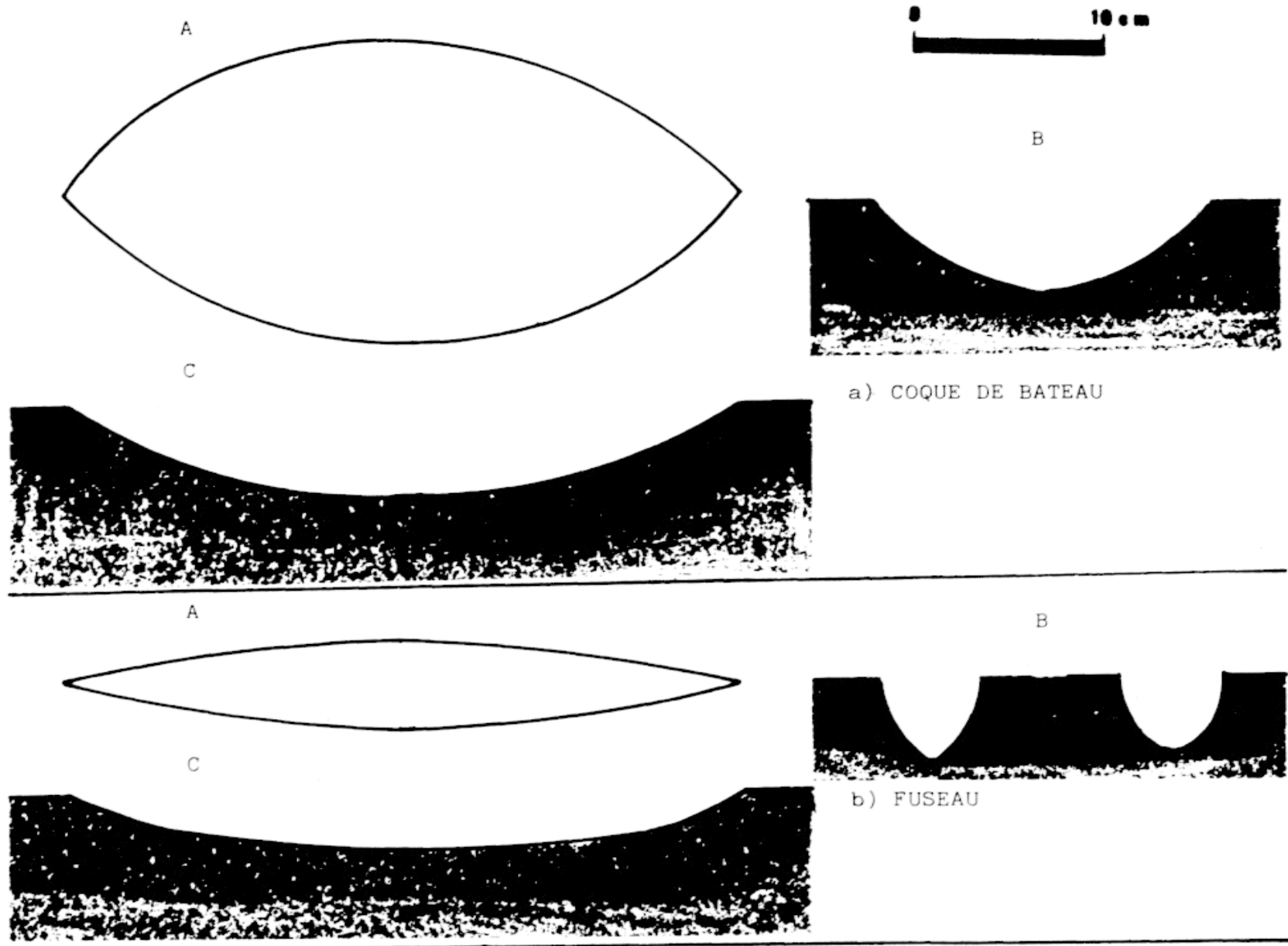

b) FUSEAL
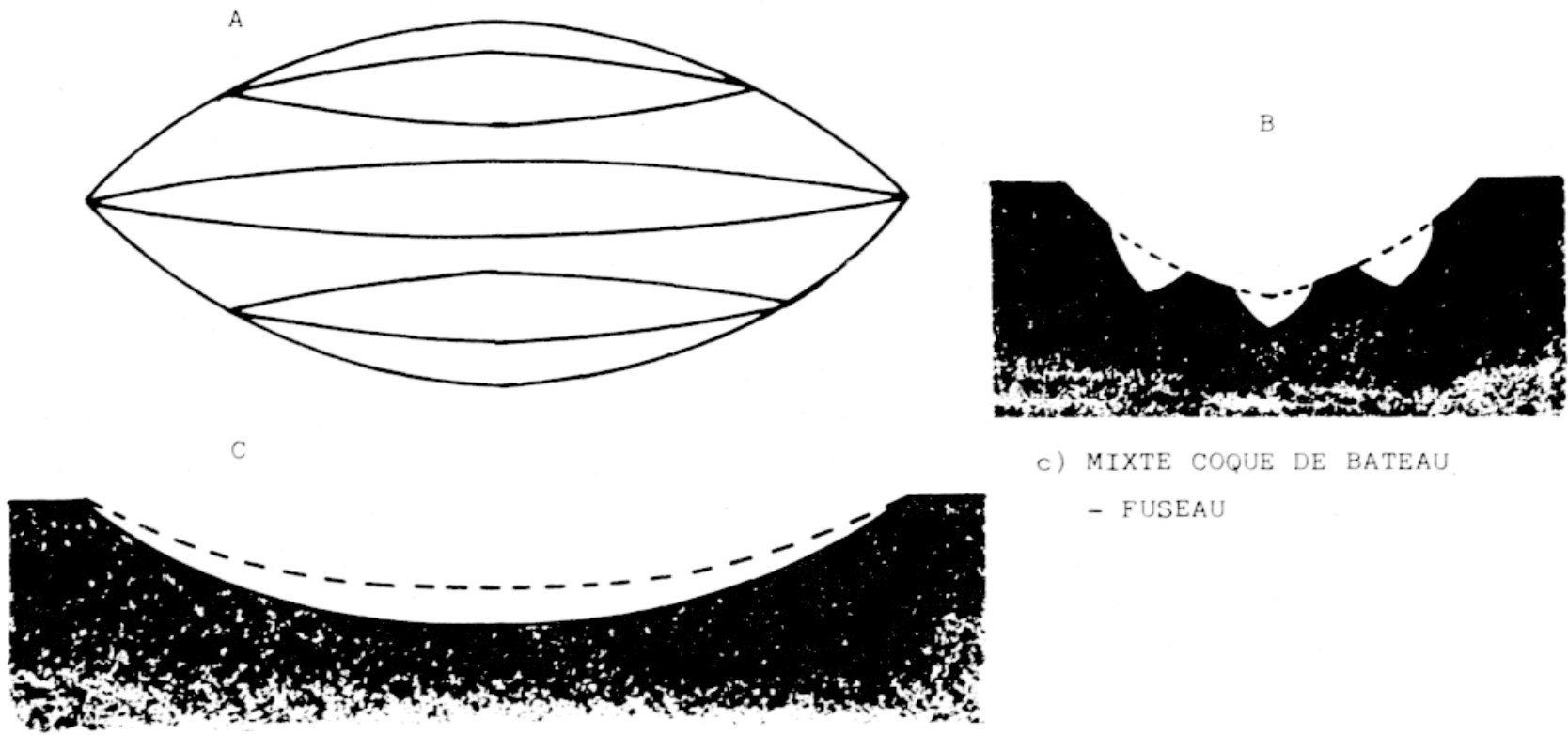

c) MIXTE COQUE DE BATEAU

- FUSEAU

Fig. 4. - Les trois types principaux de polissoirs découverts dans les sites. A : plan; B : section transversale; $\mathrm{C}$ : section longitudinale. 
comme dans l'île de Cayenne par exemple. Pourtant cette dernière ainsi que les îlets de Rémire présentent, avec les galets de leurs torrents (celui du Rorota) remaniés et bien aplatis par les grosses mers (diorite, dolérite, amphibolite) des échantillons parfaitement utilisables. J. C. Bubberman (1972) signale que, de nos jours, les Akurio du sud-est du Surinam préfèrent de très loin la source de Litany pour chercher leurs pierres.

Archéologiquement, ce type de commerce semble être attesté par la découverte au pied du saut Mapaou (bas Approuague) d'un lot de 36 pierres juste dégrossies et taillées en forme de haches (figure 8c). On peut imaginer qu'une pirogue, emportant ces cailloux destinés à être polis - et peut-être échangés - se soit renversée au passage de ce rapide particulièrement dangereux. Les roches étaient donc soigneusement choisies et, faisant l'objet de commerce, avaient une valeur non négligeable dans les relations inter-tribales. Il est possible que cette " course au gisement " ait provoqué des tensions et des guerres entre groupes pour l'occupation d'un territoire, mais ceci n'est encore nullement attesté.

\section{Le façonnage de la lame}

Jusqu'à présent, peu de haches en pierre taillée ont été retrouvées en Guyane française (seulement quatre spécimens connus), l'ensemble du matériel lithique étant poli.

La fabrication d'une lame devait débuter par la mise en forme de la pierre : elle aurait été taillée par petites percussions jusqu'à obtention de la forme désirée. L'objet était ensuite poli (on peut imaginer que c'est en observant les galets polis naturellement dans les torrents, que naquit l'idée du polissage des outils). Le polissage (percussion oblique-punctiforme) consiste à user le caillou dans une masse rocheuse compacte à l'aide de sable comme abrasif et d'eau comme délayant, ce qui explique la localisation des rochers à polissoirs sur les plages du littoral et le bord des rivières. Le polissage s'effectuait par frottement de la pierre sur le rocher dans un mouvement rotatif ou alternatif rectiligne (va-et-vient). Les polissoirs sont les traces d'usure laissées sur la roche par ce travail.

Plusieurs formes de polissoirs sont reconnues, allongées ou circulaires, et déterminées par la partie de l'objet que l'on travaillait : longue et étroite dite " en fuseau ", pour les côtés et le talon; ronde ou ovale, appelée " en cupule " et " en coque de bateau ", pour les faces et pour l'aiguisage du tranchant. Cinq types morphologiques de polissoirs sont différenciés : en cupule, en cupule à protubérance, en coque de bateau, en amande et en fuseau. Il arrive parfois que des polissoirs en fuseau soient faits dans le fond de coques de bateau, en raison du manque de place sur le rocher, ou parce qu'il était plus simple de travailler sur une surface déjà lisse ; on parle alors de polissoir mixte (figure 4). Les mêmes formes de polissoirs se retrouvent dans les autres Guyanes et dans certaines îles des Antilles.

Le polissoir agit à la fois comme un moule et comme une meule. Certaines propriétés pétrographiques sont alors exigées. La compacité et l'homogénéité d'abord, puis la dureté, sont fournies par les diorites plus ou moins quartzifères, par certains faciès migmatiques (par exemple, les gneiss de la série de l'île de Cayenne), et par les granites dont les affleurements constituent une bonne moitié 

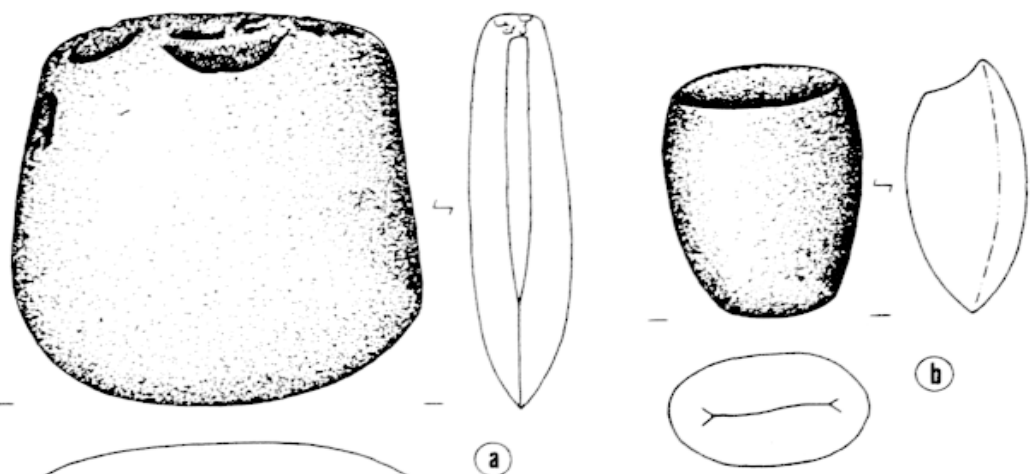

(b)

(a)
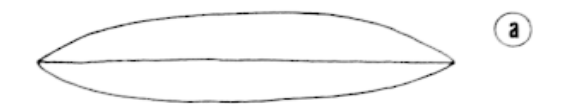

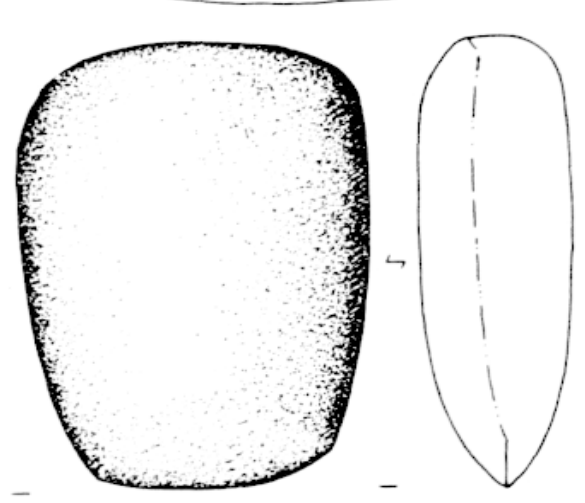

(c)
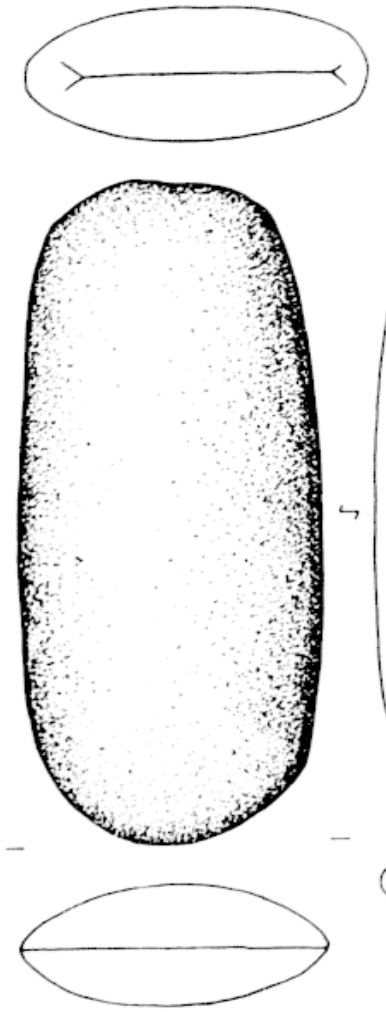

(4)
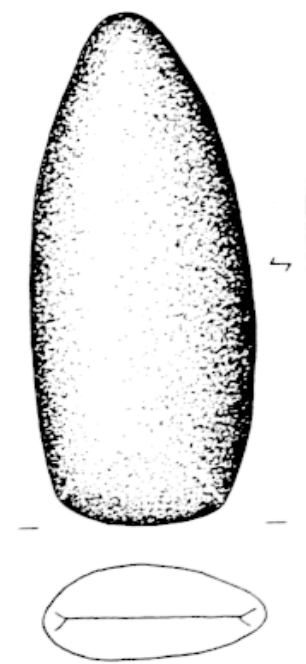

(d)
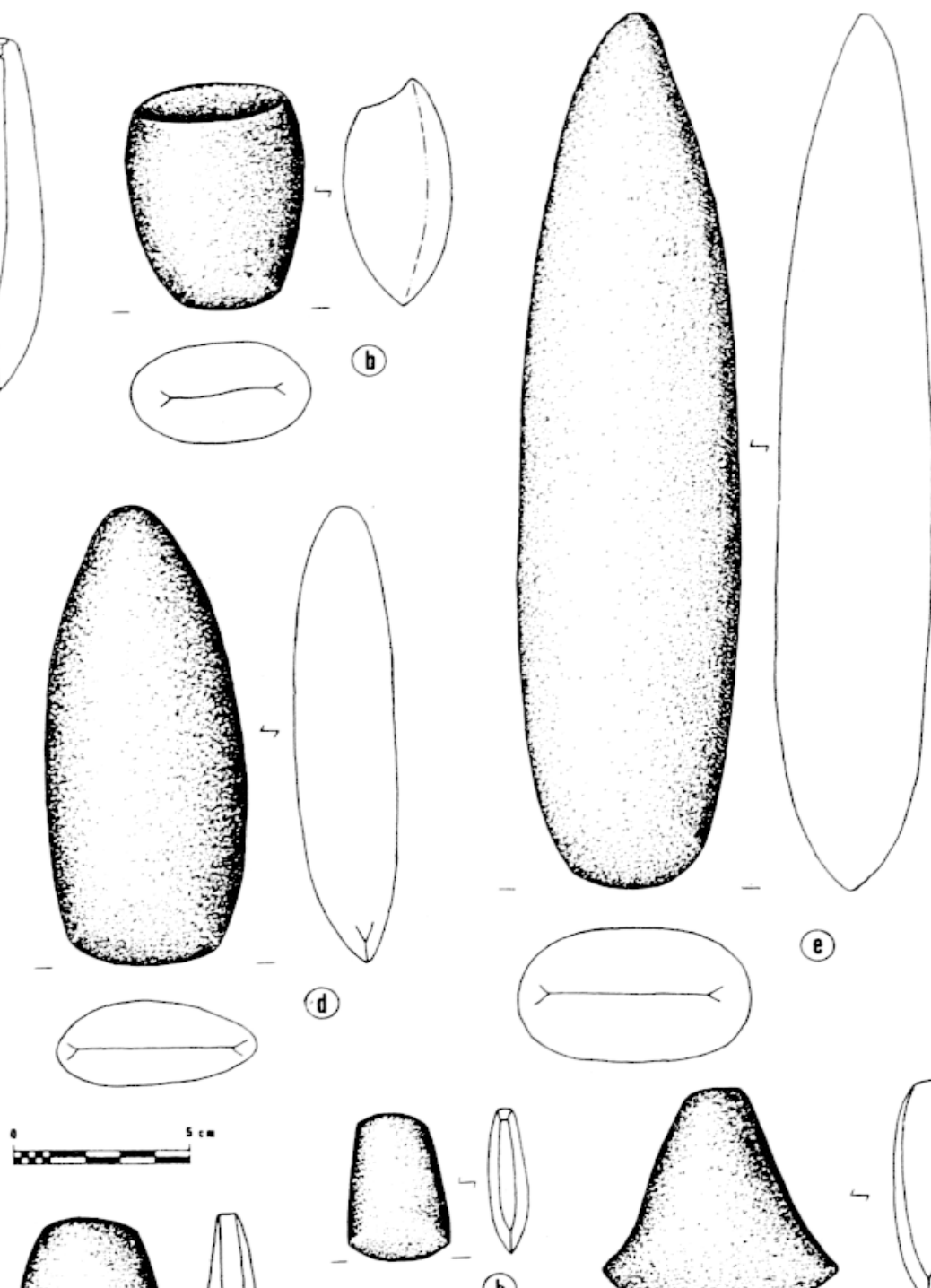

(h)

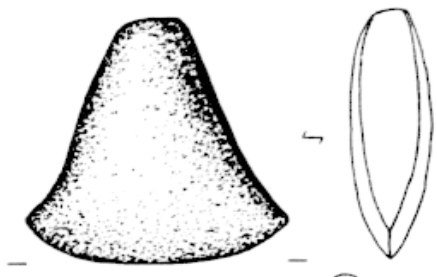

(i)
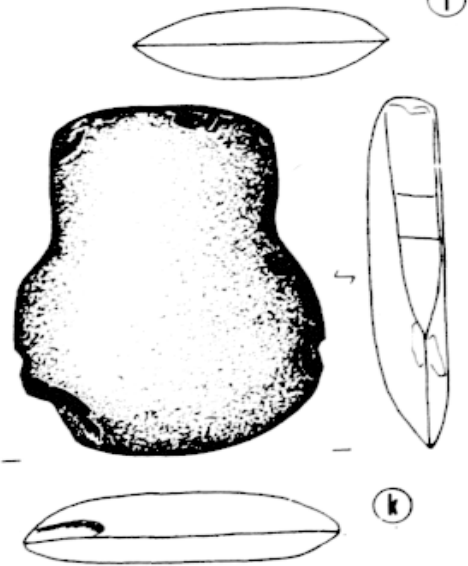

(a)

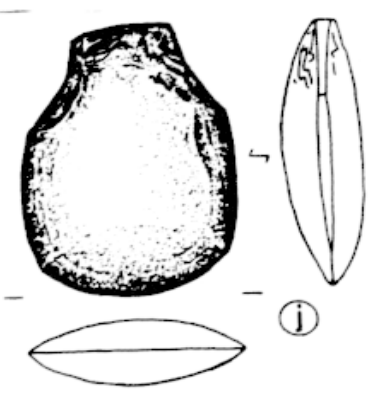

FIG. 5. - Différents types de " haches simples ». Chaque figure présente une vue de face, une vue de profil, et une vue du tranchant. a) Type Alb (BU6, Matarony); b) Ala (EL9, Courcibo); c) Ala (AX14, Matarony); d) A2b (BU5, Matarony); e) A2a (EL17, Sinnamary); I A lb (AX22, Matarony); g) Ala (AC30, Impératrice); h) Alc (BU12, Matarony); i) A3a (AG17, Maroni); j) A Sa (EL12, Sinnamary); k) A5b (AX4, Matarony). 

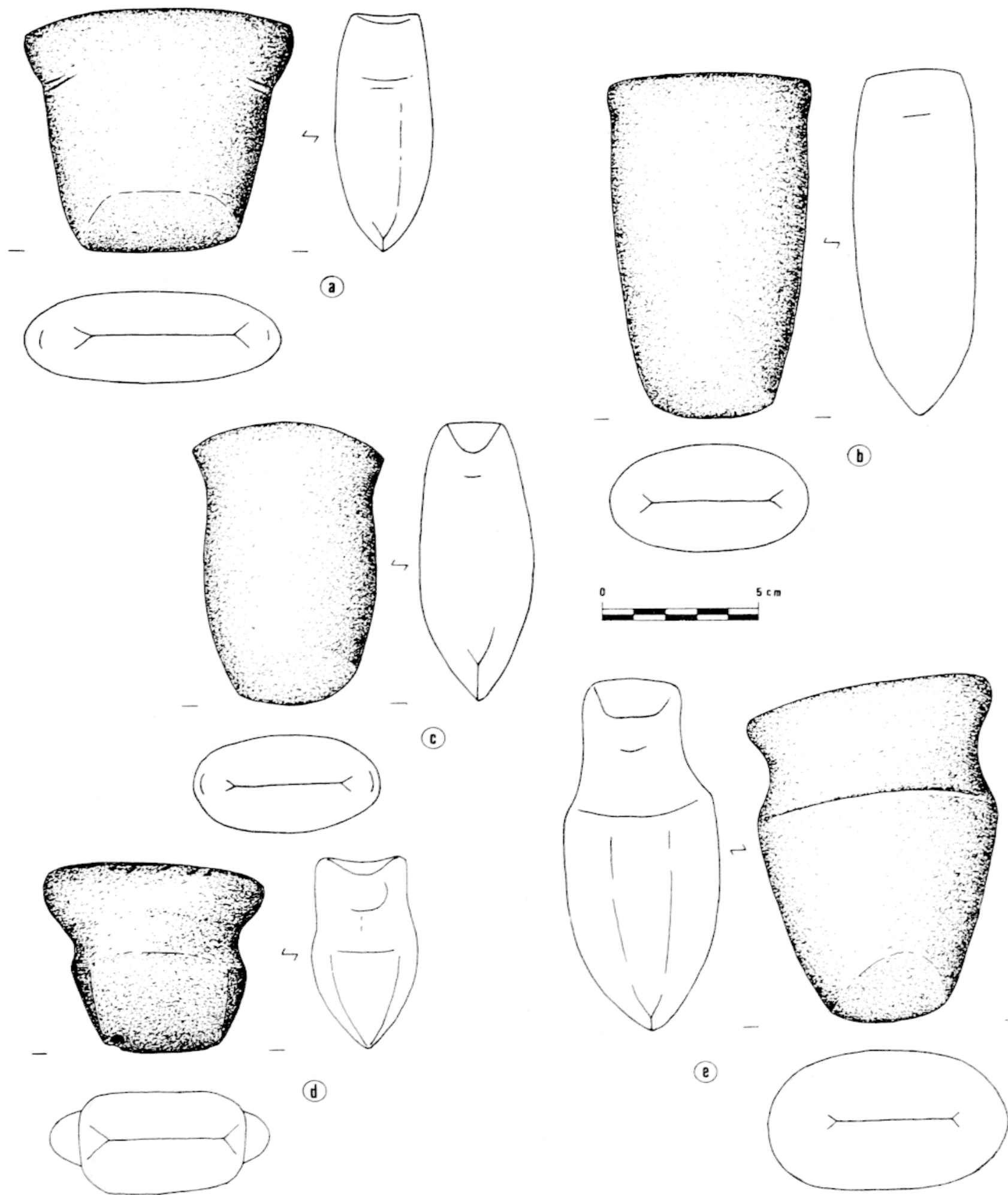

FiG. 6. - Différentes formes de " haches à oreilles ". a) Type Bla (St1, Île de Cayenne): b) Blaa (EL10, Sinnamary); c) Blaa (AX7, Matarony); d) B1c (PH6, Matarony); e) BId (MU4, localisation inconnue). 

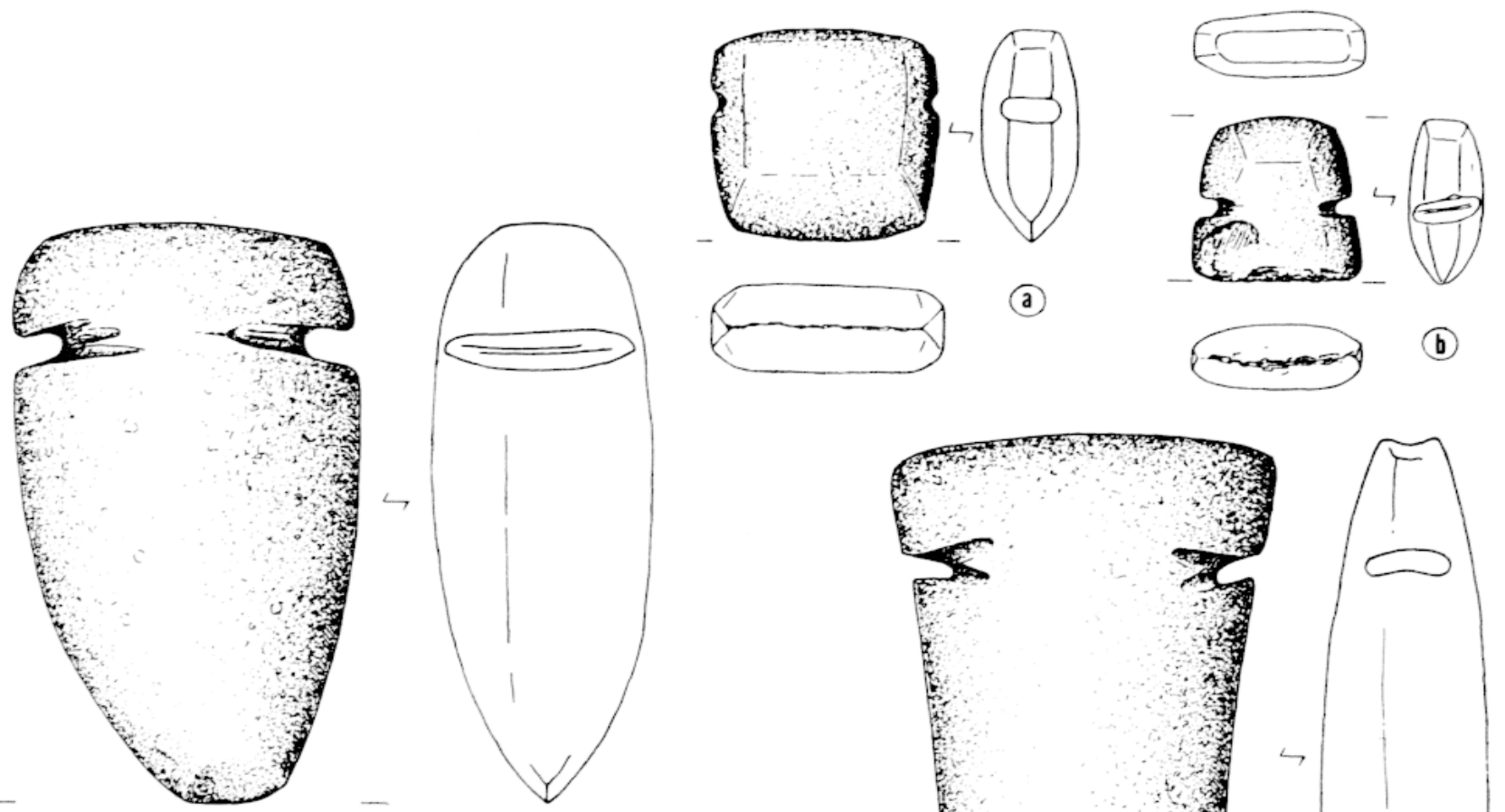

(a)
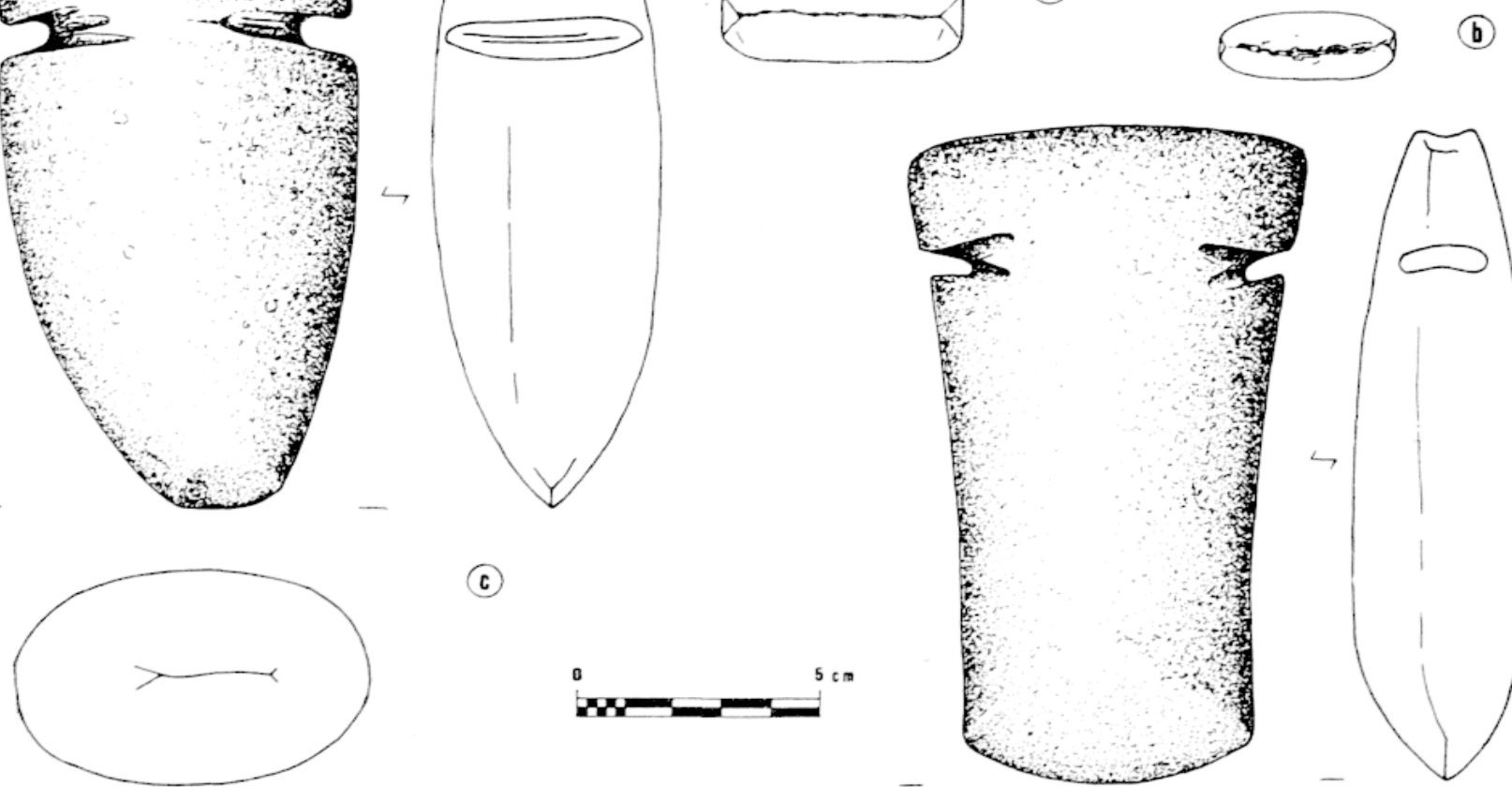

(c)
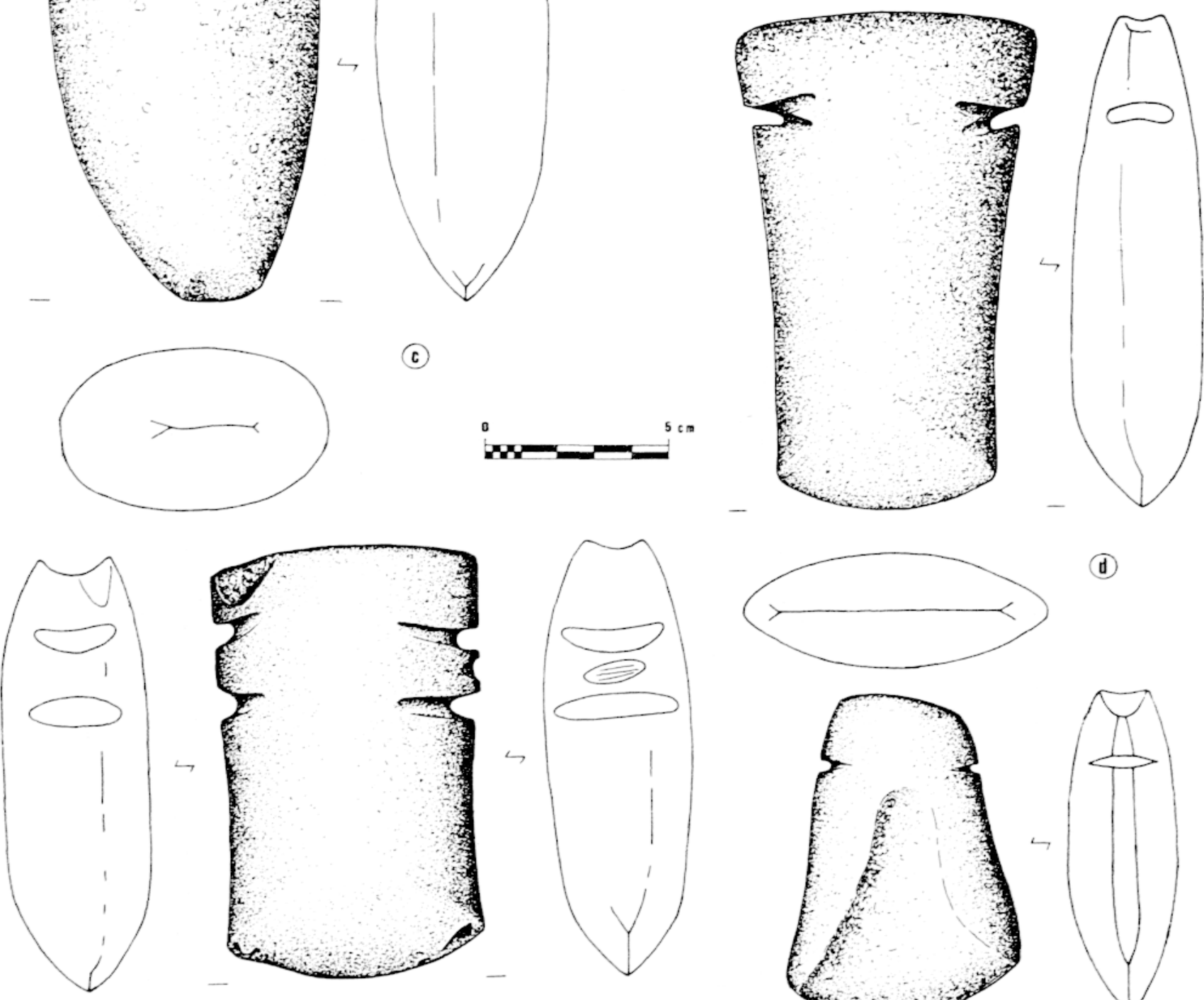

(d)

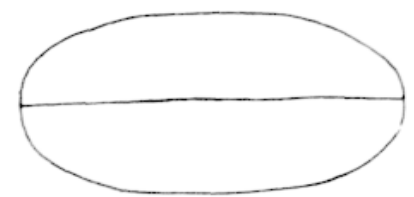

(e)

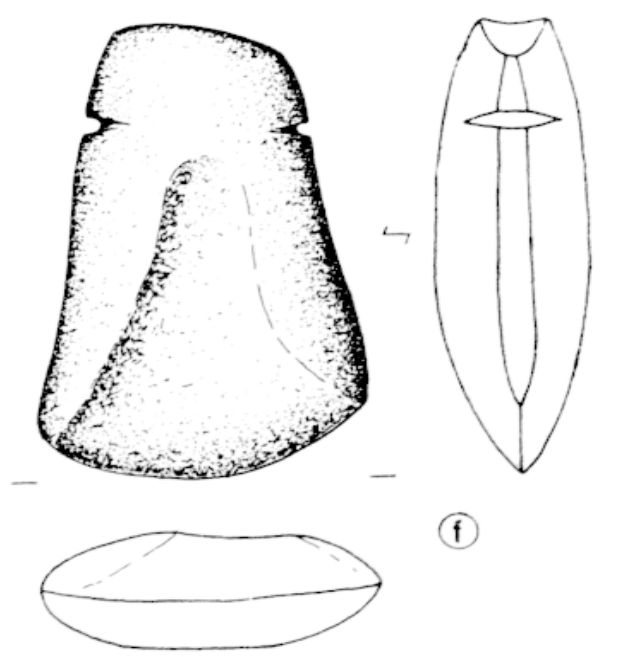

Fig. 7. - Différentes formes de " haches à encoches ". a) Type Cla (Pa2, Île de Cayenne); b) Cla (HU37, Île de Cayenne); c) C2al (TN2, Approuague); d) Cla (JA1, Maroni); e) C2al (AX2, Matarony); f) C2bl (BAl, Saül). 


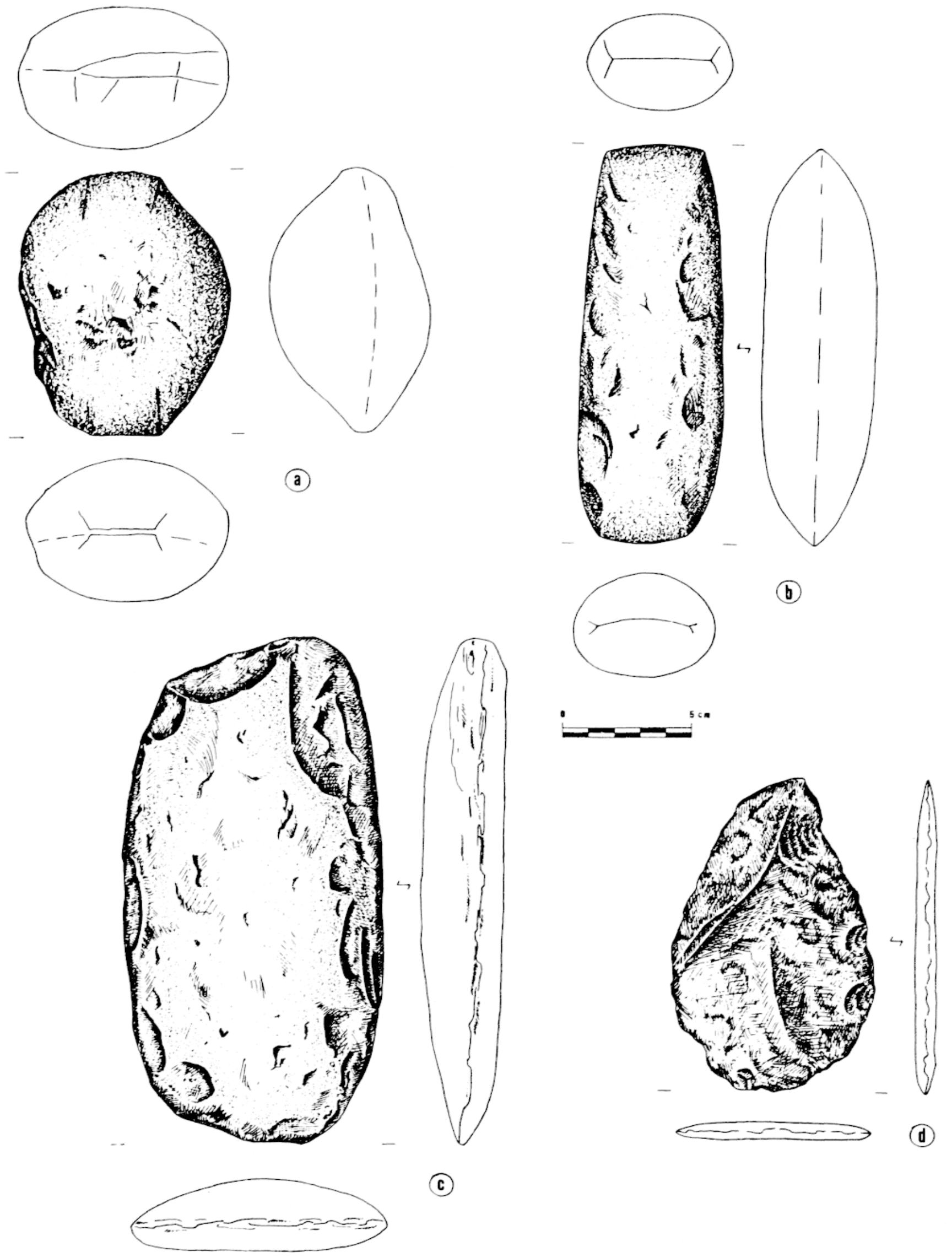

FIG. 8. - Différentes formes de haches à double tranchant et de " haches taillées ".

- Haches à double tranchant : a) Forme 2 (YA2, Macouria); b) Forme 1 (EL3, Courcibo).

- "Haches taillées ": c) avec début de polissage (AC2O, Approuague); d) haches taillée très fine (AL11, Approuague). 
du socle guyanais. Ces roches à gros cristaux durs permettent la première attaque de l'ébauche par un puissant effet de rabotage. La mise en forme de l'objet commence ensuite dans le polissoir dont le volume approche le plus la forme désirée. A la manière d'une marmite d'abrasion, le moule va alors devenir une meule, et ce grâce au sable. Les sables anguleux et non usés (ou N.U. pour les géomorphologues) des rivières doivent avoir, au départ, une efficacité supérieure à celle des sables émoussés luisants (E.L.) du bord de mer. Mais la fracturation rapide des grains de sable du littoral, par effet de pression de l'abrasion, aboutit vite à une mouture agressive. Cependant, celle-ci finit vite par se pulvériser en un " lait " rendu boueux et coloré par les produits d'usure de l'objet façonné. L'eau retient l'abrasif en suspension; elle permet l'évacuation des moutures fines. Le dosage d'eau et de sable est important pour le degré de façonnage désiré : un matelas abrasif efficace permet la fracturation des grains superficiels du sable, tout en épargnant le plus possible le polissoir lui-même : celui-ci ne joue qu'un rôle d'encadrement et de mise en forme. Le son peut être un indice non négligeable, pour l'artisan, de la donne interaction entre le polissoir-moule et le sable-meule.

Le polissage fin et l'aiguisage seront obtenus de préférence par des polissoirs sur dolérites: ces roches filoniennes compactes, cohérentes, dures et fraîches, retiennent bien l'empreinte des angles ou des bordures courbes des polissoirs. Elles peuvent prendre un poli assez doux et, sable exclu, avec de l'eau - voire de la vase sur la côte (qui jouerait le rôle de l'huile dans l'affutage des outils modernes en fer) - elles assurent une finition satisfaisante du poli des outils. Constitués de tufs cinéritiques, ceux-ci seront dans l'ensemble bien moins durs que la dolérite.

Pour faire les encoches sur les haches, il suffisait de frotter une liane ou une baguette de bois dur, avec ou sans sable, afin d'user la pierre.

Chez les Akurio, le polissage est limité au tranchant de la lame, dont le degré d'aiguisage est déterminé par une pression du bord de la pierre contre la langue. Ils peuvent fabriquer une hache avec son manche en une journée (Bubberman 1972). Les Akurio utilisent des polissoirs fixes sur les rochers des criques, mais également des polissoirs mobiles qu'ils tiennent étroitement entre leurs pieds pendant le travail.

Plus de 130 rochers à polissoirs sont actuellement répertoriés en Guyane française, situés aussi bien dans l'intérieur du territoire que sur le littoral. Ils sont souvent couverts de nombreux polissoirs de différents types. Néanmoins, un repérage précis et une étude poussée de ces sites sont nécessaires, afin d'établir une carte de la distribution des polissoirs de Guyane française. A partir de cela, on pourrait éventuellement déduire les zones d'implantation humaine privilégiées du territoire.

De nouveaux essais expérimentaux de polissage sont encore à faire afin de mieux connaitre la technique de travail, et notamment pour comprendre la méthode de façonnage des pièces de forme élaborée comme des haches à oreilles, les haches à tranchant étendu ou les haches à gorge. 


\section{L'emmanchement de la lame}

La plupart des haches et herminettes paraissent avoir été fixées dans un manche en bois. Les récits de quelques anciens explorateurs et la découverte de lames encore emmanchées, permettent de saisir quelques méthodes utilisées pour fixer la pierre au bois. Deux techniques principales sont ainsi attestées pour l'emmanchement des pierres : la fixation par inclusion et la fixation par attache.

L'emmanchement par inclusion. Pour inclure une lame dans un bois, on creusait dans une branche un trou la traversant complètement ou en partie seulement. Bubberman (1972) précise que la cavité était creusée avec une dent d'agouti aiguisée, mais l'on pouvait probablement utiliser aussi un petit burin de pierre, un coquillage, etc., et même le feu, pour faciliter le travail. S'il était plus aisé de façonner un bois tendre, celui-ci n'en était pas plus intéressant pour autant, dans la mesure où l'on obtenait ainsi un manche fragile, utilisable peu de temps.

Les manches de haches qui ont été retrouvés sont tous faits d'un bois dur - et l'on a évoqué un bois du genre Duroia pour les manches des deux haches emmanchées retrouvées dans l'Approuague - mais cela ne constitue évidemment pas une preuve suffisante, les manches de bois tendre n'ayant certainement pas pu se conserver jusqu'à nos jours. Actuellement, certains groupes amérindiens d'Amazonie utilisent des écorces souples comme manches temporaires pour leurs lames de haches en pierre.

La pierre était ensuite placée dans la cavité et, comme le montrent les lames de certaines haches (notamment celles encore emmanchées du saut Mapaou), il était parfois nécessaire de retailler un peu le talon pour l'ajuster au trou. La hache pétaloïde pouvait se contenter de ce système, grâce à sa forme effilée qui la retenait dans la cognée qu'elle traversait de part en part. Une hache de ce type a été retrouvée au Guyana (figure 9c). Mais dans la plupart des autres cas, un renforcement de la fixation était indispensable. Du latex (résine) ou du goudron pouvait être introduit dans la cavité afin de coller la pierre. Geijskes témoigne de l'usage de balata pour combler le trou trop grand (figures 9a et b).Des lianes végétales aidaient à maintenir la pierre en place (figure 10d) ou resserraient la cognée pour éviter que le bois se fende (figures $10 \mathrm{a}, \mathrm{b}$, e et $\mathrm{f}$ ). Cette dernière méthode est courante chez les Akurio, bien qu'ils possèdent aussi des haches dont la lame est simplement enfoncée dans le manche. Également, une hache provenant des Ararandeuara du Brésil est emmanchée avec une consolidation faite de lianes. Enfin, on utilisait parfois des liens et du mastic à la fois pour renforcer l'emmanchement (figure 10d).

Barrère décrit, en 1743, les haches en pierre polie des Galibi : elles étaient enfoncées dans un bois très dur et maintenues par du fil de pitte (Kuraua) et de la résine de many (Karrimanni) qui servait de goudron. En 1924, Walter Roth signale l'existence de spécimens très rares où la lame est emboîtée dans un manche de bois de section rectangulaire qui rappelle les massues sans lame des Galibi des XVIII $^{\mathbf{e}}$ et $\mathrm{XIX}^{\mathrm{e}}$ siècles. Charles H. De Goeje (1955), à partir des données de l'expédition d'Albrinck de 1938, décrit les haches en pierre des Trio rencontrées dans la forêt surinamienne : la lame était attachée dans le trou de la cognée avec une corde dont le nœud était si solide qu'il ne pouvait être défait. Le manche, d'environ $30 \mathrm{~cm}$ de long, était rond dans sa partie supérieure et s'amincissait vers la poignée pour finir 


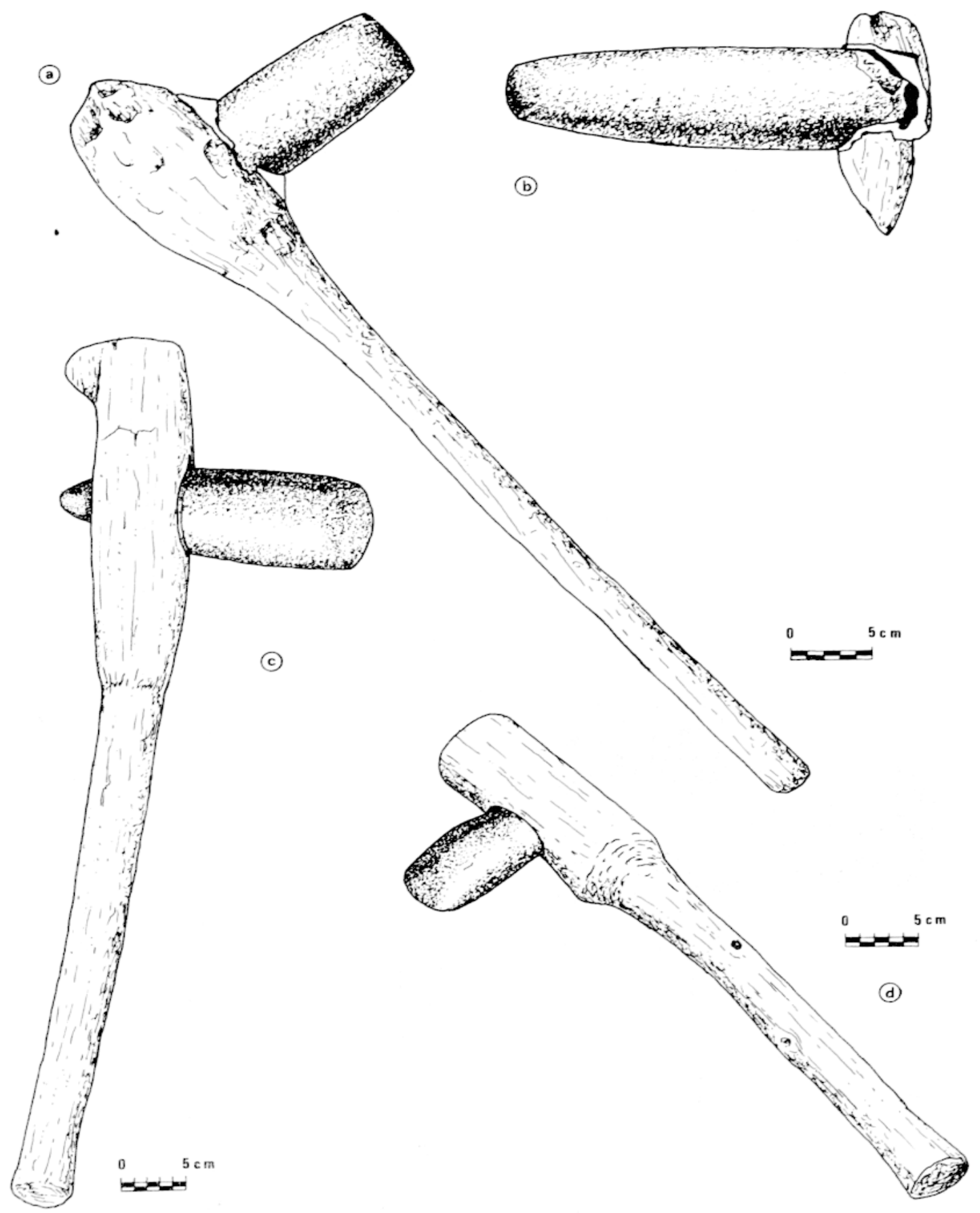

FIG. 9. - Les emmanchements par inclusion.

a et b) Haches emmanchées découvertes à Saut Mapaou (bas Approuague) en 1883. Pierre (tuf andésitique) enchâssée dans un bois dur (Duroïa) avec du latex. Collection AGAE.

a) Manche de $64 \mathrm{~cm}$ de long daté au C14 par le CNRS (Laboratoire de Gif-sur-Yvette) de $1480-1600$ aptès $J$.C.

b) Traces d'usure de liens sur le bois.

c) Hache emmanchée trouvée dans la rivière Mazaruni (Guyana) en 1974. Le manche mesure $65 \mathrm{~cm}$ de long. Collection Ashton France (d'après photo de Dennis Williams, 1978).

d) Hache emmanchée des Indiens Tapahùunas, rio Arinos (Brésil). Le manche mesure $47,5 \mathrm{~cm}$ de long. Rapportée par Lauro Sodré en 1897. Museo Emilio Goeldi, Belèm (d'après photo du catalogue). 

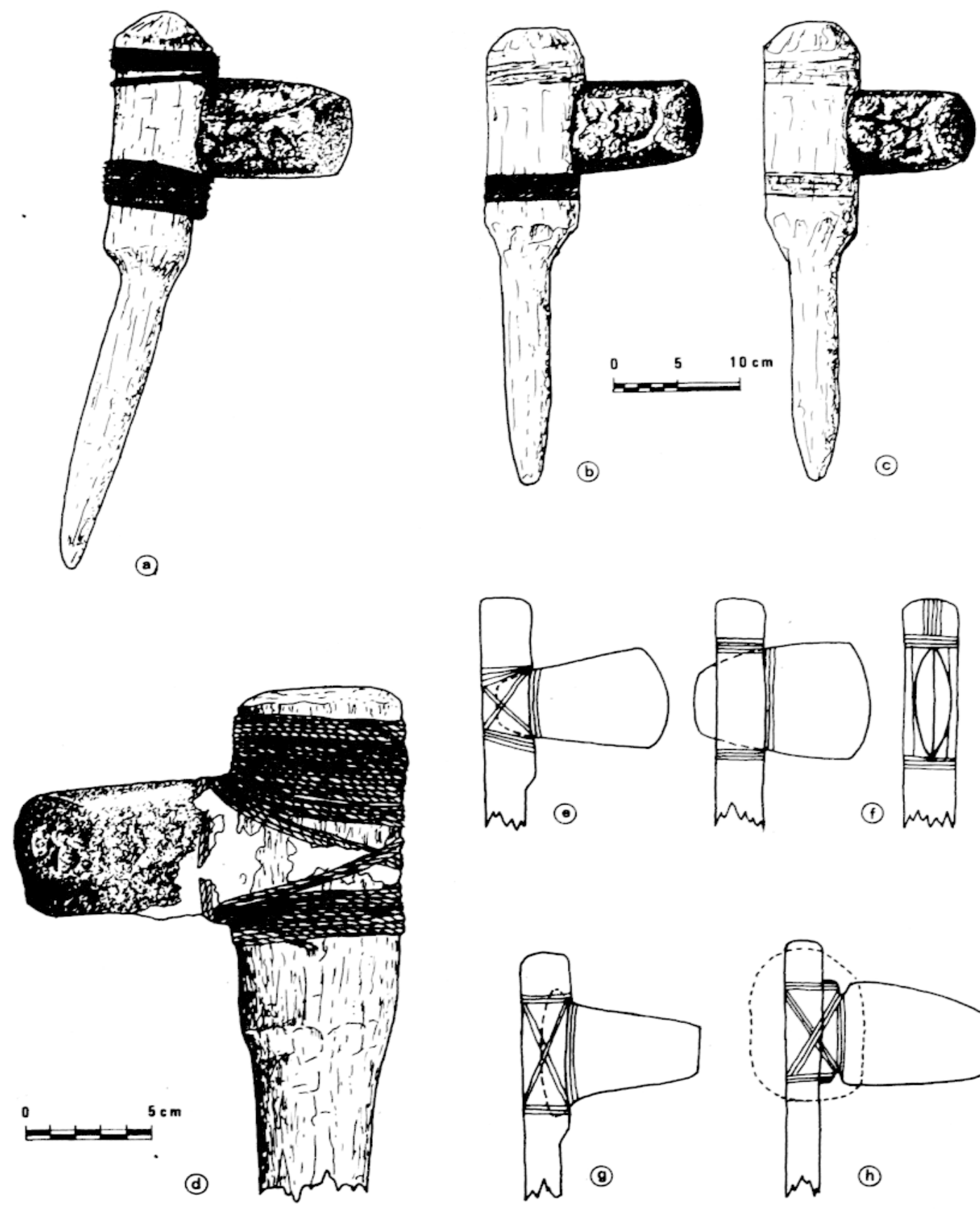

FIG. 10. - Les emmanchements àl'aide de ligatures.

a) Hache Akurio (Surinam) rapportée en 1968 (d'après photo de F. C. Bubberman, 1972). Dimensions inconnues.

b et c) Haches Akurios échangées par André Cognat (Antecume Pata) dans la région du haut Maroni (d'après photos de Jean-Luc Chodkiewicz).

d) Hache Akurio emmanchée par inclusion avec des liens et du latex, provenant du sud-est du Surinam. Lame en métadolérite. Collection du Surinam Museum, Paramaribo (d'après photo de Aad Boomert, 1977).

e et $\mathrm{f}$ ) " Deux modes de ligature de haches en pierre " (d'après Pierre Grenand, 1982, figure 13, page 189).

$\mathrm{g}$ et $\mathrm{h})$ Hypothèses d'emmanchement par ligature.

g) Inclusion et liens.

h) Liens et gangue de résine et de terre. 

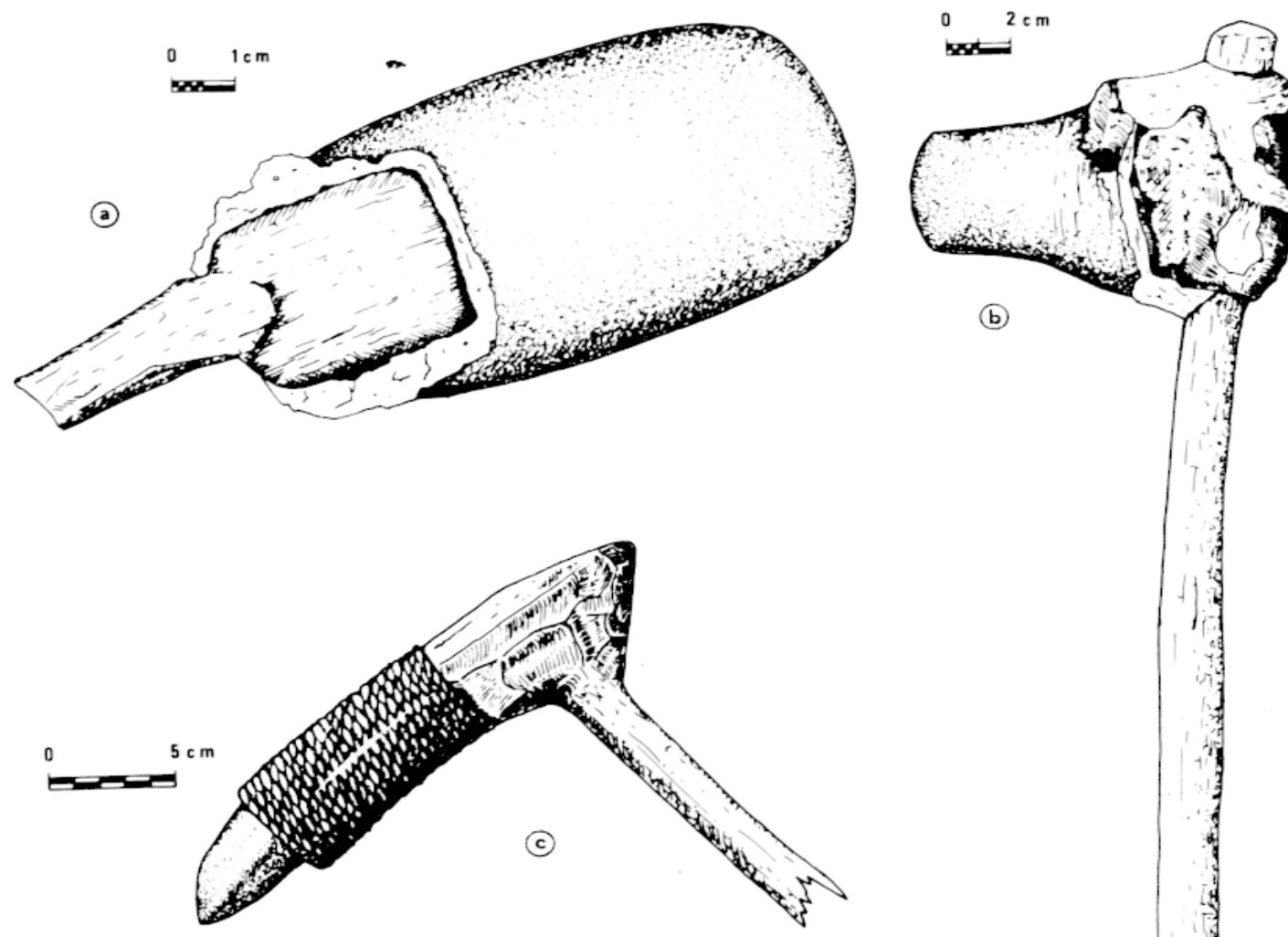

(b)

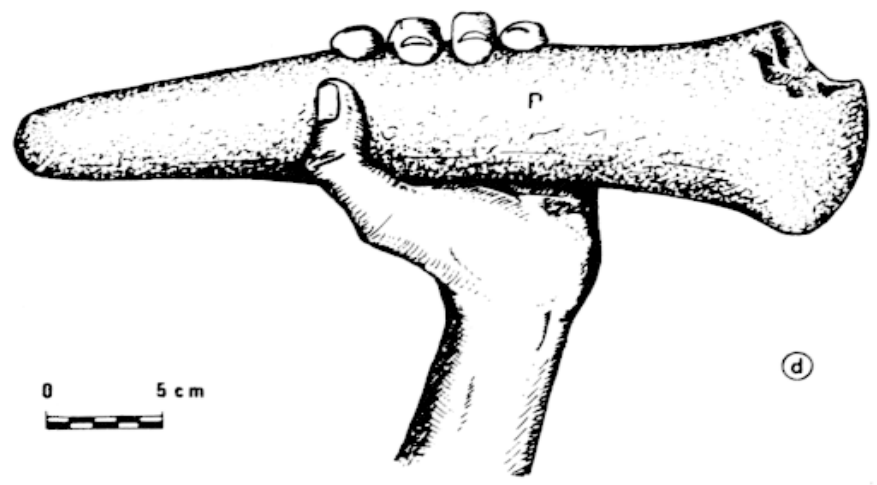

FIG. 11. - Diverses méthodes d'emmanchement.

a) Herminette emmanchée découverte en 1984 dans la crique Matarony (bas Approuague). La pierre est coincée dans une fourche de bois à l'aide de latex. Collection privée (d'après informations et photo d'Alain Cornette et d'Yves Wack, 1985).

b) Hache à oreilles emmanchée retrouvée dans la crique Matarony (bas Approuague) en 1984. La lame est maintenue dans un manchon de résine, de cire, de gomme et d'argile. Le manche mesure $58 \mathrm{~cm}$ de long. Collection privée (d'après informations et photos d'Alain Cornette et d'Yves Wack, 1985).

c) Méthode d'emmanchement d'une herminette en pierre en Papouasie (Nouvelle-Guinée) qui aurait pu être utilisée dans les Guyanes.

d) Hypothèse de préhension directe avec la main d'une hache à double tranchant provenant de la crique Leblond (Sinnamary, EL6). La lame mesure $36 \mathrm{~cm}$ de long. Collection privée. 
en pointe obtuse. Les Akurio de la région du haut Maroni et du haut Tapanahony utilisent encore des haches emmanchées identiques (figures 10a, b, c et d).

En Guyane française, six haches emmanchées par inclusion ont été à ce jour découvertes. En 1983, un chercheur d'or a récupéré, dans la suceuse de sa drague, à Saut Mapaou, dans le lit de l'Approuague, deux haches emmanchées par inclusion à l'aide de latex. Il les a vendues par la suite à l'Association Guyanaise d'Archéologie et d'Ethnographie. L'une des haches a son manche entier, mesurant $64 \mathrm{~cm}$ de long (figure 9a). Après quelques retouches sur le talon, la pierre a été introduite dans la cavité préalablement enduite de résine, puis la cognée a été taillée.

On s'est interrogé sur l'éventualité d'une inclusion de la lame dans un arbre en pleine croissance, mais, après observation, des botanistes de l'ORSTOM de Cayenne estiment que, pour deux raisons, cette hypothèse doit être rejetée : tout d'abord la présence de latex à l'intérieur de la cavité prouve que la sève ne circulait plus lors de l'inclusion, par conséquent que le bois était mort. Enfin, et surtout, les cernes de croissance de l'arbre ne contournent pas la lame, ce qui signifie que celuici n'a donc pas continué son évolution après l'enchâssage de la hache. Si en effet un corps étranger, comme une pierre, est introduit dans un arbre vivant les cernes de croissance contourneront ensuite l'intrus, tout comme ils le feraient d'une branche.

Le manche complet de la hache a été daté au Carbone 14 par le Centre de Faibles Radioactivités du laboratoire CNRS-CEA de Gif-sur-Yvette : il remonterait à 1480-1600 après J.-C. Le résultat de cette datation est intéressant, car c'est à cette époque que les Amérindiens du littoral (Saut Mapaou, dernier rapide où la marée se fait sentir, marque la frontière entre le littoral et l'intérieur) ont abandonné leurs haches en pierre pour leur préférer celles en métal, apportées par les Européens qui commençaient à envahir le territoire. Le bassin de l'Approuague était, à l'époque de la conquête, principalement le domaine des Nourague (ou Norak), groupe de langue Tupi (Pères J. Grillet et F. Béchamal 1674).

De nos jours, il semble que quelques groupes d'Akurio (Acooreo au sud Surinam selon Harcourt en 1613, mais on cite aussi les Acouri, les Akulijo, les Akuri ou les Akuriyo), isolés et sans contact avec la civilisation européenne, utilisent encore des haches en pierre. Selon plusieurs auteurs, les Akurio du haut Oulémali et haut Litany (frontière Guyane française - Surinar $r_{\star}$ ) ont une vie entièrement nomadique depuis une centaine d'années. Ils auraient été jadis des agriculteurs en contact avec les Européens. Une fois cette relation perdue, ils n'auraient plus disposé d'outils en fer, et auraient été contraints de recommencer la fabrication des haches en pierre. Aad Boomert (1977) suggère que leurs haches, n'ayant que le tranchant poli, révèleraient une décadence technique, un oubli technologique. Findlay a vu ces haches avec des pierres provenant de la crique Alapari. En 1968, André Cognat, Wayana d'adoption, a échangé avec des Akurio plusieurs haches en pierre emmanchées (figures $10 \mathrm{~b}$ et c). Les haches Akurio connues sont toutes très semblables : une poignée en bois d'une longueur variant autour de $35-40 \mathrm{~cm}$ et effilée à sa base, la partie cylindrique supportant la pierre plus épaisse et parfois enserrée par des liens végétaux qui empêchent le bois de se fendre.

L'emmanchement des herminettes en pierre est encore très mal connu. Un seul 
exemplaire encore emmanché a pour l'instant été découvert. Il a été retrouvé en 1985 par un orpailleur, dans le lit de la crique Matarony (bas Approuague). La lame est encastrée dans le même axe qu'un manche en bois se terminant en fourche et dont il ne reste qu'un fragment. Un amalgame de résine et d'argile maintient la pierre dans son logement (figure 11a). Cette pièce unique est particulièrement intéressante car c'est le premier spécimen d'herminette emmanchée trouvé dans les Guyanes. A défaut de documents, on peut proposer d'autres méthodes d'emmanchement des herminettes en Guyane : notamment la pierre pouvait être enchâssée dans un manche coudé à l'aide de liens végétaux comme cela se pratique dans plusieurs parties du monde (Océanie, Afrique) - (figure 11c).

Certains textes anciens signalent la méthode de l'inclusion de lame dans un arbre vivant, qui se développait alors en se soudant à la pierre. En 1796, Stedman explique comment les Amérindiens qu'il a rencontrés au Surinam emmanchaient leurs haches : la pierre était placée dans un arbre en croissance et s'y attachait très intimement, puis le bois était coupé pour être façonné.

Dans son ouvrage sur les Amériques (1883), Jules Crevaux reprendra la description de ce procédé, sans pour autant l'avoir observé personnellement. En 1948, Lalung écrit qu'il fallait attendre deux à trois ans pour que la pierre se soude à l'arbre; cela semble long, si c'est vraiment le cas, il est compréhensible que cette méthode ait été peu utilisée. Jusqu'à présent, aucune découverte de hache emmanchée de cette façon ne permet de prouver la pratique de cette technique dans les Guyanes; néanmoins, il est probable que certains groupes amérindiens l'ont utilisée. Par ailleurs, ce système est attesté dans certaines autres régions du globe; Daniel Alexandre, botaniste à l'ORSTOM, a observé ce type de haches en Côte d'Ivoire, et des employés du CTFT lui ont décrit des arbres vivants dans lesquels des pierres étaient enchâssées.

L'emmanchement par attache. La seconde méthode d'emmanchement, par attache, est moins bien connue. La pierre était appliquée sur une tige de bois et attachée à celle-ci par des liens végétaux qui passaient dans les encoches ou derrière les oreilles. La rainure transversale que l'on voit sur le talon de certaines de ces haches, s'adaptait à l'arrondi de la cognée et évitait que la lame ait du jeu. En 1946, Henry et Paule Reichlen décrivent cette méthode et rapportent la découverte sur la rivière Ucayali au Pérou de trois haches attachées avec des fibres végétales. Lorsqu'il n'y a pas de rainure, deux petits bâtonnets auraient maintenu la pierre. H. et P. Reichlen signalent trois haches de ce type, dont une provient des Huachipairi du rio Beni en Bolivie, et les deux autres des Guarayo. Il est également possible qu'une petite partie du talon ait été enfoncée dans le manche puis liée : il s'ag:rait alors d'un compromis entre la méthode par inclusion et celle par attache (figure $10 \mathrm{~g}$ ). Il semble que la pierre ait aussi été parfois fixée grâce à un amalgame d'argile, de cire et de résine, comme le montre une hache retrouvée dans la crique Matarony (figure 11b). Cette hache, ainsi que l'herminette emmanchée provenant du même endroit, sont actuellement en dépôt pour étude au Centre National de la Recherche Archéologique Sub-aquatique d'Annecy. L'analyse précise des différents composants du manchon permettrait de mieux comprendre ce type de fixation qui apparaît, de prime abord, peu fiable. $H$. et $P$. Reichlen rapportent également la trouvaille, sur le rio Napo (Équateur-Brésil) d'une hache fixée à l'aide d'une gangue de cire. 
Les haches à main. Toutes les haches n'étaient probablement pas emmanchées. dans un bois, mais pouvaient être tenues directement à la main. Ainsi, certaines haches à oreilles et haches à double tranchant semblent bien adaptées à ce type de prise. On peut citer notamment une longue et lourde hache à double tranchant (EL 6) provenant de la crique Leblond (Sinnamary), qui paraît difficilement emmanchable et qui semblerait plutôt destinée à être tenue à la main (figure 11d).

\section{LA FONCTION DES HACHES ET DES HERMINETTES EN PIERRE}

On peut distinguer deux utilisations majeures de ces outils : le travail du bois et l'armement.

\section{Le travail du bois}

Comme le souligne Charles $H$. De Goeje, pour la vie en forêt, la hache de pierre était aussi indispensable à l'Amérindien d'autrefois que l'est aujourd'hui le sabre pour les Noirs Réfugiés, c'est-à-dire "toto-aki-topo".

Il existe au moins trois fonctions principales : l'abattage des arbres, la fabrication des pirogues et la construction des carbets (cases amérindiennes sans murs).

Le bûcheronnage. La plupart des Amérindiens de Guyane française menaient autrefois une vie semi-nomade de chasseur-cueilleur pratiquant l'agriculture. Ils auraient vécu par groupes de 50 à 100 personnes et déplaçaient leur village au bout d'un certain laps de temps, allant de quatre à huit ans, lorsque leur aire d'approvisionnement était épuisée ou que le toit de palmes des carbets était pourri. Pour installer leur village et leur abattis, il leur fallait donc ouvrir une clairière dans la forêt.

Si l'on utilisait parfois le feu pour faire mourir les gros arbres, il n'en était pas moins nécessaire de couper les petits arbustes et certains arbres qu'on ne brûlait pas. Charles H. de Goeje (1955) décrit une méthode qui était utilisée pour abattre les arbres chez les Amérindiens : travaillant à deux, on entoure le tronc d'une corde placée à environ un mètre du sol de façon à ce que chacun coupe à la même hauteur, et l'on taille à peu près un tiers de l'épaisseur du tronc, que l'on fait rompre ensuite. Bubberman (1972) montre que les Akurio utilisent leurs haches pour fendre le bois : les petits arbres sont coupés en tous sens puis rompus à la main,tandis que les arbres plus importants sont d'abord brûlés à la base du tronc puis, une fois carbonisés, abattus à l'aide de la hache. Le même auteur signale que, pour les Akurio, la hache sert essentiellement à leur quête inlassable de miel sauvage, part importante de leur nourriture. Ils l'utilisent pour agrandir les trous d'arbres dans lesquels nichent les abeilles.

La fabrication des pirogues. Un des moyens privilégiés de circulation dans l'épaisse forêt tropicale est la navigation par voie fluviale. Les Amérindiens construisent donc depuis longtemps des pirogues. Il en existe de différentes sortes : 
les petites pirogues individuelles en écorce, de plus importantes destinées au transport de matériel et de personnes, et enfin, pour la mer, de très grandes pirogues éventuellement munies de voiles.

Le R.P. Barbotin a étudié, en 1973, le matériel lithique de l'île caraïbe de Marie-Galante, en tentant d'établir un parallèle avec les témoignages des chroniqueurs anciens tels que le Père Raymond Breton (1635). Bien que son travail porte sur des pièces différentes de celles étudiées ici, et davantage adaptées à la vie insulaire, il demeure intéressant pour la compréhension de la fabrication des pirogues. Ainsi, souligne-t-il, le " travail " - la notion européenne de contrainte qu'implique ce terme étant très éloignée de la conception qu'en ont les Amérindiens - le plus fastidieux et le plus long (estimé à un an compte tenu des autres activités indispensables à la survie du groupe) était la construction des pirogues de haute mer, qui pouvaient contenir chacune de 60 à 60 personnes et étaient destinées à les emmener chaque année visiter d'autres groupes en Guyane.

il fallait en effet, pour construire de telles pirogues, abattre un gros arbre, le creuser en utilisant feu et herminettes, découper les deux longues planches destinées à rehausser les plat-bords et, enfin, confectionner l'étoupe.

Le R.P. Barbotin a essayé de retrouver l'utilisation des différents outils pour chacune de ces étapes. Les Karib du littoral des Guyanes réalisaient probablement le même type de pirogue pour la mer, car différents témoignages montrent qu'ils naviguaient aussi sur de grandes distances et qu'il leur était plus courant de voyager le long de la côte entre les bouches de l'Orénoque et de l'Amazone, que de s'enfoncer dans l'intérieur du pays. Alexis Tiouka (communication personnelle) signale que, de nos jours, les Galibi de la région de Mana construisent une pirogue d'une douzaine de mètres en un an environ, ce qui correspond, compte tenu des autres activités quotidiennes, à trois mois de travail continu.

Pour les petites pirogues en écorce, on découpait avec une hache deux plaques d'écorce que l'on assemblait ensuite à l'aide de cordes. Dans l'ouvrage de Jules Crevaux (1887), une gravure montre cette activité chez les Boni.

La construction des carbets. Les Amérindiens des Guyanes vivent cases sans murs, simplement composées de poteaux de bois enfoncés dans le sol et tenus généralement entre eux par un système d'emboîtement consolidé par des lianes. L'ensemble est recouvert d'un toit de palmes tressées. De la coupe et l'élaguage des grosses branches qui deviendront poteaux et traverses, jusqu'à la taille des palmes du toit, la hache est donc un outil indispensable pour la construction du carbet.

\section{L'armement}

Apparemment, certaines haches étaient spécifiquement destinées à servir d'armes. La hache est un instrument maniable, rapide et efficace pour le combat au corps à corps. Elle peut être utilisée pour achever un animal blessé, mais aussi pour la guerre. On connaît 4 autres types d'armes amérindiennes dans les Guyanes : les arcs et flèches ainsi que les lances dont les pointes sont faites avec un bois très dur; les harpons munis de nos jours d'une pointe en métal, mais qui probablement était autrefois en pierre ou en os; enfin les massues (à section quadrangulaire chez les Galibi). 
Yves d'Évreux rapporte un précieux témoignage de son voyage à Maranhão au nord du Brésil (aujourd'hui Sao Luis) en 1613-1614:

Cette carcasse vivante (un Amérindien survivant d'une bataille) enseigna le mieux qu'il put le lieu où gisaient ses compagnons morts : on les trouva la tête fendue, les haches de pierre dont on leur avait fendu la tête étaient posées sur leur corps car c'est leur coutume de ne plus se servir d'une arme quand avec elle ils ont tué un de leurs ennemis. (...) Carouatapyran m'apprit ce que je ne savais pas en touchant ces haches, faites d'une pierre très dure et taillées en forme de croissant, car il me dit que tous les mois au premier jour du croissant, les Tremembais avaient coutume de veiller toute la nuit pour faire ces haches et qu'ils ne cessaient qu'elles ne fussent parfaites, ayant cette superstition que s'ils portaient ces haches en guerre, ils n'étaient jamais vaincus mais remportaient la victoire sur leurs ennemis. Pendant qu'ils font ces haches, les femmes, filles et enfants sont au dehors des ajoupaves (cases), dansant et chantant à la face du croissant (Yves d'Évreux, réédition 1985, page 138).

Ce type de hache " en demi-lune " n'a pour l'instant pas été retrouvé en Guyane française, mais il en a été découvert un exemplaire au Guyana et deux autres encore emmanchés au Brésil, dont un qui est en possession du Museo Emilio Goeldi de Belém. Ryden (1937) pense qu'il s'agissait tout d'abord d'une arme mais que peu à peu associée à des notions magiques, elle se transforma en emblème de chef, c'est-à-dire en arme cérémonielle.

Jules Crevaux raconte également le massacre d'un groupe de Boni par une centaine de Wayana armés de haches en pierre.

L'attitude défensive avec la hache brandie des Akurio rencontrés par Albrinck en 1938 montre bien l'utilisation guerrière de ces instruments. Bubberman (1972) signale aussi l'anecdote, survenue en 1968, d'un groupe d'Akurio qui détruisirent à coups de haches en pierre le moteur d'un voyageur trop curieux.

Toutes les haches de guerre n'affectaient probablement pas toujours une forme de croissant, mais elles devaient parfois être moins élaborées. En observant le poids et la morphologie de la hache emmanchée entière de saut Mapaou, on s'aperçoit qu'elle est tout à fait inadaptée au travail du bois : trop lourde et trop fine pour que le manche résiste longtemps à des chocs répétés contre un obstacle dur. En revanche, elle semble pouvoir être très efficace utilisée comme masse maniée à deux mains : son poids lui confère une grande "force de frappe " et sa forme la rend très maniable. Il est donc probable qu'il s'agit ici d'une hache de guerre et non pas d'un outil de travail forestier.

\section{Les autres fonctions}

Certaines haches de formes particulières avaient probablement un but spécifique. Ainsi, les haches sculptées, gravées ou de très petite taille et de forme élaborée ont pu être conçues pour une fonction de parure, notamment pour être portées en pendentif autour du cou. Peut-être avaient-elles un rôle de distinction sociale. D'autres ont certainement eu une signification magico-religieuse, soit portées en amulette, soit en tant qu'objet rituel durant des cérémonies. Enfin, des haches miniatures ont pu également servir d'offrandes funéraires. 
Il semble que les haches en pierre soient des outils essentiellement masculins. Tout d'abord, leurs diverses utilisations concernent davantage les activités de l'homme que celles des femmes. Jean-Luc Chodkiewicz (communication personnelle) signale, d'après un informateur Wayana, que lorsqu'une femme Akurio emprunte la hache de son époux pour un quelconque travail; une fois celui-ci achevé, elle s'empresse de la rendre à son "propriétaire ". Le récit d'Yves d'Évreux est également intéressant à ce sujet, puisqu'il montre que les hommes polissent les haches en demi-lune pendant que les femmes et les enfants, exclus de cette activité, dansent et chantent. Malgré cela, il serait encore imprudent d'avancer des théories sur la hache comme symbole de division sexuelle.

\section{LA RÉPARTITION GÉOGRAPHIQUE DES HACHES ET DES HERMINETTES}

Les différents inventaires de haches en pierre polie qui ont été faits sur les Guyanes confirment les constatations de $\mathrm{H}$. et P. Reichlen. Les haches simples sont plus fréquentes en Amazonie et dans les Guyanes, mais les types simples ont une forme universelle et sont donc peu utilisables pour effectuer des comparaisons.

Selon le Dr. Rivet, la hache à encoches est un outil de type essentiellement amazonien. On le retrouve jusqu'aux Andes, mais sa fréquence d'apparition s'étend surtout vers les Guyanes et, de là, sur les Antilles. Le même auteur pensait que les haches dont les oreilles forment un angle droit proviendraient des vallées interandines et de la côte Pacifique, pour pénétrer ultérieurement vers l'est du continent.

En conclusion de son article, Aad Boomert (1979) propose une distribution géographique de ses différents types de haches. Il pense que certaines formes de lames seraient typiquement antillaises (A2a, A3b) tandis que d'autres seraient d'origine amazonienne (B1a, Cla, C2al), bien que certains autres types encore de haches se retrouvent autant dans l'aire antillaise que dans l'aire amazonienne (les Guyanes comprises). Aad Boomert voit là une preuve d'influence culturelle directe des Guyanes sur l'aire antillaise. Certaines haches sont seulement connues dans les Guyanes et pourraient représenter des formes locales : A4, B1c, Bid et B2b au Guyana; A5a, A 5b, C2ab et $\mathrm{C} 2 \mathrm{~b} 2$ en Guyane française et, pour l'ensemble des Guyanes, B2a et C2b1.

La forme A3 peut être considérée comme une copie des hachettes en fer européennes, introduites dans les Guyanes au XVII ${ }^{\mathbf{e}}$ siècle. On ne la retrouve qu'en Guyane française (AG 17 provenant du bas Maroni, figure 5) et dans le sud du Surinam. Les Amérindiens ont pu les exécuter après avoir vu des hommes se servir de haches en métal, mais peut-être n'avaient-elles qu'un rôle cérémoniel ou symbolique. A. Boomert pense que quelques formes au moins de haches à double tranchant du Guyana et du Surinam auraient été importées de l'est du Vénézuela aux temps historiques.

Aad Boomert termine son article en remarquant que :

L'ensemble des types de haches des Guyanes illustre parfaitement la position géographique de la région à l'époque précolombienne; étroitement liée à 
l'Amazonie du nord mais reliée au bord oriental de l'aire caraïbe. Les caractéristiques d'Amazonie surpassent celles des Antilles dans les Guyanes. La plupart des haches distribuées aux Antilles et en Amazonie se sont probablement d'abord développées sur l'Amazone. Mais le manque de recherches archéologiques empêche l'identification d'un commerce direct des haches (Aad Boomert, 1979, page 111).

Betty Meggers et Clifford Evans (1957) considèrent "l'innovation culturelle " des haches en pierre comme assez tardive. Selon eux, l'apparition de ces outils coïncide avec le début de la phase archéologique Marajoara à Marajo (4001300 ans après J.-C.) et de la phase Arùa dans le territoire de l'Amapà (1200-1800 après $\mathbf{J}$.-C.). Les haches en pierre seraient donc plutôt récentes qu'anciennes dans la séquence culturelle de l'embouchure de l'Amazone. Mais cette théorie est discutable.

Sur le territoire de la Guyane française, toute tentative de répartition géographique des haches et herminettes en pierre reste pour l'instant très aléatoire. En effet, sur les 239 pièces localisées, peu ont été découvertes dans des sites archéologiques fouillés (seulement 37 , soit $15 \%$ du total). La majorité des outils provenait du lit d'une rivière, car la plupart des collections se composaient de pièces récoltées au même endroit, dans la drague d'un orpailleur. A cet égard, la carte des lieux de trouvailles de haches en pierre de Guyane française s'adapte assez bien à la carte de localisation de certaines concessions aurifères du pays. $\mathrm{Ce}$ fait devrait inciter les archéologues à s'intéresser aux découvertes des chercheurs d'or en ayant soin de recueillir le maximum d'informations sur les emplacements, la profondeur dans les alluvions, les objets associés, etc...

Mais pour l'instant, qu'il s'agisse de la Guyane française seule, ou de l'ensemble des Guyanes, il est trop tôt pour établir une carte de répartition des outils en pierre ou pour en désigner les lieux d'origine. Avant d'avancer des conclusions, il faudrait réunir l'ensemble des données archéologiques et ethnographiques pour l'aire amazonienne et l'aire caraïbe.

\section{LES HACHES EN PIERRE DE NOS JOURS}

\section{Les derniers utilisateurs}

L'utilisation des haches en pierre polie a rapidement disparu après l'arrivée des Européens. En effet il semble que les premiers produits échangés aient été les hachettes en fer et les perles de verre. Les Pères J. Grillet et F. Béchamel (1674), rapportant leur remontée de l'Approuague, évoquent leur chargement de haches en fer destinées à être échangées avec les Amérindiens. Dans certaines grottes funéraires de la baie d'Oyapock, on a retrouvé des perles en verre datables de l'époque du contact grâce au matériel céramique de style Aristé et, pour le site de Trou Reliquaire, par la datation au $\mathrm{C} 14$ d'échantillons de charbon de bois, indiquant 1360-1480 de notre ère. Probablement parce que les outils en fer étaient 
très prisés par les Amérindiens, les mentions d'usage d'instruments en pierre chez ceux-ci se font très rares à partir des $\mathrm{XVII}^{-\mathrm{XVIII}^{\mathrm{e}}}$ siècles dans les récits de missionnaires et d'explorateurs.

Néanmoins, plusieurs personnes ont rencontré ces dernières années de petits groupes d'Amérindiens se servant encore d'outils en pierre, par exemple les Akurio du sud du Surinam. Au demeurant, ces derniers échangent assez facilement leur hache en pierre contre un sabre ou une hache de métal (Bubberman 1972 et André Cognat en 1968).

Pierre Grenand rapporte le témoignage d'un Amérindien de Camopi, sur l'Oyapock qui a vu utiliser des haches en pierre dans cette région:

Un informateur wayapi-puku, Tawika, l'a vu utiliser dans la région de l'Amapari, il y a une trentaine d'années. Conséquemment, le mode de montage des pierres de haches polies (par enchâssage dans un bois creusé ou par ligature sur un bois encoché) et l'utilisation des polissoirs sont connus. Il semble que la technique se soit maintenue de loin en loin, concurrencée par les outils en fer, jusqu'au début du XIX ${ }^{e}$ siècle pour les Wayapi du centre et du nord, et jusque vers 1940 pour les Wawapi-puku (Pierre Grenand 1982: 191).

\section{Un culte pour les haches}

Au début du siècle, Paul Sangnier a noté que les Wayana du Litany conservaient les pierres de haches qu'ils trouvaient, les considérant comme des "Pierres à Tonnerre ", et qu'ils en faisaient des imitations en céramique sans usage pratique. Certains fragments étaient même utilisés pour l'aiguisage des outils en fer.

La croyance s'attachant aux «Pierres à tonnerre" semble en fait trouver son origine chez les populations noires. Les lames de hache en pierre polie sont en effet considérées comme des objets divins par les créoles : pendant les orages, des pierres tomberaient du ciel, provoquant les éclairs et le tonnerre par le choc de la chute, et elles fendraient les arbres en enflammant leurs feuillages. Il est possible de la retrouver si elle n'est pas descendue trop profondément dans le sol, et on la conservera alors précieusement, car elle est censée apporter de la chance à son possesseur. Cette croyance demeure toujours très vivace en Guyane, mais elle l'est encore davantage dans certaines îles des Caraïbes. Jesse (1973) a étudié ce phénomène dans ces îles : à Trinidad, à Sainte-Lucie et en Haïti, de véritables cultes sont voués à ces haches. Elles sont vénérées au même titre que les anges et les saints car, provenant du ciel, elles sont des envois de Dieu. Pour néanmoins vérifier l'authenticité d'une pierre ainsi trouvée, on la suspend par un fil de coton audessus d'un feu -- bougie ou charbons ardents. S'il s'agit d'une " pierre ", le fil ne brûlera pas. Elles sont par la suite conservées dans des chapelles et gardées par des prêtres et serviteurs, sont régulièrement " nourries" avec de l'huile et/ou le sang d'animaux sacrifiés. On leur attribue des dons relevant du surnaturel : elles peuvent transpirer, siffler, parler, se déplacer seules ou parfois même se changer en serpent. Leurs pouvoirs, symbolique et de guérison, sont très importants car assimilés à ceux de Shango, Dieu du tonnerre et de la foudre, qu'elles représentent. Ce culte, et jusque dans ses formes rituelles, est très proche de ceux voués aux 
pierres de haches en Afrique où elles sont le symbole et l'instrument du même Dieu Shango, qui a le pouvoir d'envoyer la pluie et de favoriser les récoltes.

Ayant rapporté plusieurs témoignages allant dans ce sens, Jesse associe le culte du Dieu Shango à celui d'Afrique de l'Ouest. Il aurait été importé par les premiers esclaves qui, trouvant dans les champs des pierres polies similaires à celles de leur pays, leur auraient porté la vénération et consacré les rituels qui leur étaient familiers.

Bien que de telles pratiques ne soient pas attestées dans les Guyanes, les croyances sur les pouvoirs protecteurs des pierres polies y sont très fortes ainsi qu'au Brésil, à Cuba et en Jamaïque. Il faut également remarquer que ce genre de croyances est assez répandu à travers le monde. L'Abbé Breuil n'a-t-il pas été un des premiers à s'intéresser aux "pierres-tonnerres" jonchant le sol de France?

\section{REMERCIEMENTS}

Nous remercions vivement Marc Lointier, hydrologue à l'ORSTOM de Cayenne, pour sa collaboration à l'étude statistique et pour son aide logistique.

Nous exprimons également notre gratitude à Aad Boomert, à Denis Williams et au R.P. Maurice Barbotin pour la documentation qu'ils nous ont fournie. Roger Plat, du Service des Mines de Cayenne, a eu l'amabilité de déterminer la pétrographie des roches; les botanistes de l'ORSTOM de Cayenne nous ont donné des précisions utiles; Jany Sauvanet a mis à notre disposition ses talents de photographe. Les corrections de Pierre Grenand ainsi que celles de Catherine Reynaud nous furent précieuses.

Enfin, cet article n'aurait jamais vu le jour sans la gentillesse des personnes qui nous ont laissé étudier leurs collections d'outils en pierre amérindiens et sans les nombreux amis qui nous ont prêté des documents.

\section{BIBLIOGRAPHIE}

Abonnenc, E., 1952. "Inventaire et distribution des sites archéologiques de Guyane française ". Journal de la Société des Américanistes, nouvelle série, n 41, 43-63, Paris.

BARBotin, R.P. M., 1971. "Archéologie caraibe et chroniqueurs ». Bulletin de la Société d'Histoire de la Guadeloupe, $\mathrm{n}^{\circ} 15 / 16,53-67$.

Barrère, P., 1743. Nouvelle relation de la France Équinoxiale. Piget-Damonneville, Durand, Paris.

BOOMERT, A., 1979. "The prehistoric stone axes of the Guianas : a typological classification ". Journal of the Walter Roth Museum of Archaeology and Anthropology, volume 2, $\mathrm{n}^{\circ} 2$, 99-124, Georgetown.

BoOmert, A., KroOnenberg, S. B., 1977. " Manufacture and trade of stones artifacts in Prehistoric Surinam " dans B. L. Van Beek, R. W. Brandt and W. Groemman-Van Waateringe (eds): Ex Horreo IPP 1951-1976. Cingula 4. 9-46, Amsterdam. 
Bubberman, F. C., 1972. "Stones span the Centurie ". Surinaamse Musea, Mededelingen, $\mathrm{n}^{\circ} 9$.

Crevaux, J., 1883. Voyages dans l'Amérique du Sud. Hachette, Paris.

DE Geoje, C. H., 1955. "Les Indiens Néolithiques (avec les données de l'expédition d'Albrinck de 1938) ", Philosophie, Initiation et Mythes des Indiens de la Guyane et des contrées voisines, Paris.

AUBERT DE LA RUE, E., 1948. Reconnaissance géolugique de la Guyane française méridionale. ORSTOM éd.

D’Évreux, Y., 1985. Voyage au nord du Brésil fait en 1613 et 1614. Présentation par Hélène Clastres, Payot, Paris.

Grenand, P.. 1982. Ainsi parlaient nos ancêtres. Essai d'Ethnohistoire "Wayapi ". Travaux et documents de l'ORSTOM, n 178 , ORSTOM ed., Paris.

Grillet, P. J., 1857. « Voyage des PP. Jean Grillet et François Béchamel dans l'intérieur des Guyanes en 1674 ". Mission de Cayenne et de la Guyane française, Julien, Lanier, Cosnard et $C^{\text {ie }}$ éditeurs, Paris.

Harris. P. O'B., 1983. "Antillean axes/adzes : persistence of an Archaic Tradition". Compte-rendu $d u I X^{e}$ CIECPPA, Santo Domingo, 2 au 8 août 1981. Centre de Recherches Caraïbes, Université de Montréal, 257-290.

JeSSE, C., 1973. "Pre-Columbian stone artifacts put to strange use ". Compte-rendu du IV $V^{e}$ CIECPPA, Reduit Plage, Sainte-Lucie, 26 au 30 juillet 1971. St Lucia Archaeological and Historical Society, 68-72, Castries, St Lucia.

De Lalung, H., 1948. Les Caraibes, un peuple étrange aujourd'hui disparu. Éd. Bourrelier et $\mathrm{C}^{\mathrm{ie}}$, Paris.

Leroi-Gourhan, A., 1971. L'homme et la matière. Collection "Sciences d'aujourd'hui ", Albin Michel éd., Paris.

Meggers, B., Evans, C., 1957. Archaeological Investigations at the mouth of the Amazon. Bureau of American Ethrology, Bulletin 167, Washington.

Perriaux, 1967. Cours de sédimentologie. Faculté des Sciences, Grenoble.

Petitjean Roget, H., 1984. Rapport hache emmanchée de l'AGAE. Non publié, 11 pages et 5 figures.

, 1985. «De l'arbre à pierre verte. À propos d'une nouvelle acquisition de l'AGAE : une hache emmanchée ". Communication au $X I^{\mathrm{e}}$ Congrès International d'Archéologie de la Caraibe, Puerto Rico, 28 juillet au 3 août 1985 (sous-presse).

REICHi.eN, H. et P., 1946. "Contribution à l'archéologie de la Guyane française ". Journal de la Société des Américanistes, nouvelle série, $\mathrm{n}^{\circ}$ 35, 1-24, Paris.

Rostain, S., 1985. Rapport relatif à l'acquisition par le Conseil Régional d'une collection de pièces archéologiques guyanaises. Non publié, 14 pages.

, 1986a. Saul, rapport de mission archéologique. Non publié, 42 p., 27 fig.

..-., 1986b. État actuel de la recherche archéologique en Guyane française. En cours de publication, 63 p., 23 fig.

Rostain, S., Wack, Y., 1986. A propos des collections d'antiquités. " Rapport relatif à l'étude de quelques collections privées". Non publié, 43 p., $30 \mathrm{pl}$.

Rotr, W., 1924. "An introductory study of the arts, crafts and customs of the Guiana Indians ". 38th Annual Report of the U.S. Bureau of American Ethnology (1916-1917).

Stedman. J. G., 1978. Voyage a Surinam et dans l'intérieur de la Guiane " contenant la relation de cinq années de courses et d'observations faites dans cette contrée intéressante 
et peu connue avec des détails sur les indiens de la Guiane et les nègres ». Traduction de l'anglais par P. F. Henry, F. Buisson éd., 3 volumes, Paris.

Turenne, J. F., 1979. «Archéologie ». Atlas des départements d'outre-Mer, n 4 : la Guyane. CEGET-CNRS/ORSTOM, 1-4, pl. 17, Bordeaux/Talence.

Williams, D., 1978. "A hafted polished stone axe from the bed of the Mazaruni river". Journal of the Walter Roth Museum of Archaeology and Anthropology, vol. 1, n 1, p. 55, Georgetown. 